Article

\title{
Nonlocal Boundary Value Problems of Nonlinear Fractional $(p, q)$-Difference Equations
}

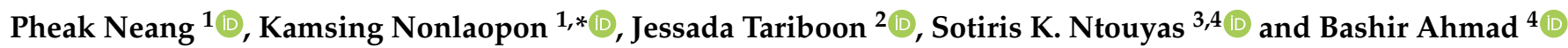 \\ 1 Department of Mathematics, Faculty of Science, Khon Kaen University, Khon Kaen 40002, Thailand; \\ pheakne@kkumail.com \\ 2 Department of Mathematics, Faculty of Applied Science, King Mongkut's University of Technology North \\ Bangkok, Bangkok 10800, Thailand; jessada.t@sci.kmutnb.ac.th \\ 3 Department of Mathematics, University of Ioannina, 45110 Ioannina, Greece; sntouyas@uoi.gr \\ 4 Nonlinear Analysis and Applied Mathematics (NAAM)-Research Group, Department of Mathematics, \\ Faculty of Science, King Abdulaziz University, P.O. Box 80203, Jeddah 21589, Saudi Arabia; \\ bashir_qau@yahoo.com \\ * Correspondence: nkamsi@kku.ac.th; Tel.: +668-6642-1582
}

check for updates

Citation: Neang, P.; Nonlaopon, K.; Tariboon, J.; Ntouyas, S.K.; Ahmad, B. Nonlocal Boundary Value Problems of Nonlinear Fractional $(p, q)$ Difference Equations. Fractal Fract. 2021, 5, 270. https://doi.org/ $10.3390 /$ fractalfract 5040270

Academic Editor: Saptarshi Das

Received: 8 November 2021

Accepted: 7 December 2021

Published: 10 December 2021

Publisher's Note: MDPI stays neutral with regard to jurisdictional claims in published maps and institutional affiliations.

Copyright: (c) 2021 by the authors. Licensee MDPI, Basel, Switzerland. This article is an open access article distributed under the terms and conditions of the Creative Commons Attribution (CC BY) license (https:// creativecommons.org/licenses/by/ $4.0 /)$.

\begin{abstract}
In this paper, we study nonlinear fractional $(p, q)$-difference equations equipped with separated nonlocal boundary conditions. The existence of solutions for the given problem is proven by applying Krasnoselskii's fixed-point theorem and the Leray-Schauder alternative. In contrast, the uniqueness of the solutions is established by employing Banach's contraction mapping principle. Examples illustrating the main results are also presented.
\end{abstract}

Keywords: Caputo fractional $(p, q)$-difference equations; nonlocal boundary conditions; existence and uniquness; Leray-Schauder alternative; fixed-point theory

MSC: 05A30; 26A51; 26D10; 26D15

\section{Introduction}

In recent years, fractional differential equations have been considered a popular field of research and have attracted many researchers' attention. This is mainly because fractional differential equations are found to be effective and more practical than classical differential equations, particularly in the mathematical modeling of dynamical systems, such as fractals and chaos. In the last two decades, the fractional differential equations have developed from theoretical aspects of the existence and uniqueness of solutions to analytic and numerical methods for finding solutions, which can be found in [1-9]. Moreover, in modern mathematical analysis, fractional differential equations have a range of applications, such as engineering and clinical disciplines, including biology, physics, chemistry, economics, signal and image processing, and control theory; see [10-17] for more details.

In 1910, F. H. Jackson initiated a study of the $q$-difference calculus or quantum calculus (briefly called $q$-calculus) in a symmetrical manner and introduced the $q$-derivative and $q$-integral, which can be found in $[18,19]$. With these results, $q$-calculus has arisen in a range of applications, such as combinatorics, orthogonal polynomials, basic hypergeometric functions, number theory, quantum theory, mechanics, and theory of relativity; see [20-40] and the references cited therein. The book by V. Kac and P. Cheung [41] covers the basic theoretical concepts of $q$-calculus.

Because the exploration has been continued by generalizing the existing results through creative discussions and novel techniques of fractional calculus, fractional $q$ difference calculus has been introduced by W.A. Al-Salam [42] and R.P. Agarwal [43]. Furthermore, fractional $q$-difference equations have attracted the attention of researchers; 
for instance, B. Ahmad et al. [44] investigated the existence results of nonlocal boundary value problems of nonlinear fractional $q$-difference equations:

$$
\left\{\begin{array}{l}
{ }^{c} D_{q}^{\alpha} x(t)=f(t, x(t)), t \in[0,1], 1<\alpha \leq 2, \\
\alpha_{1} x(0)+\beta_{1} D_{q} x(0)=\gamma_{1} x\left(\eta_{1}\right), \quad \alpha_{2} x(1)+\beta_{2} D_{q} x(1)=\gamma_{2} x\left(\eta_{2}\right),
\end{array}\right.
$$

where $f \in \mathcal{C}([0,1] \times \mathbb{R}, \mathbb{R})$ and $\alpha_{i}, \beta_{i}, \gamma_{i}, \eta_{i}(i=1,2)$ are constants with $\eta_{i} \in(0,1),{ }^{c} D_{q}^{\alpha}$ and $D_{q}$ are the fractional $q$-derivative of the Caputo type and the first-order $q$-difference operator, respectively. For more early and recent works on the existence theory of fractional $q$-difference equations, we refer to [45-48].

Later on, the subject of $(p, q)$-calculus was generalized and developed from the $q$ calculus theory into the two-parameter $(p, q)$-integer, which is used effectively in many fields, and some results on the study of $(p, q)$-calculus can be found in [49-74].

Inspired and motivated by some of the above applications, in 2018, N. Kamsrisuk et al. [75] considered the existence and uniqueness of solutions for the first-order quantum $(p, q)$-difference equation subject to a nonlocal condition:

$$
\left\{\begin{array}{l}
D_{p, q} x(t)=f(t, x(p t)), t \in[0, T / p] \\
x(0)=\alpha_{i} x(T)+\sum_{i=0}^{m} \beta_{i} \int_{0}^{\eta_{i}} x(s) d_{p_{i}, q_{i}} s
\end{array}\right.
$$

where $0<q<p \leq 1,0<q_{i}<p_{i} \leq 1(i=1,2,3, \ldots, m)$ are quantum numbers, $f \in \mathcal{C}([0, T / p] \times \mathbb{R}, \mathbb{R}), T>0, D_{p, q}$ is the $(p, q)$-difference operator, and $\alpha_{i}, \beta_{i}, \eta_{i}$ $(i=1,2, \ldots m)$ are constants.

Furthermore, C. Promsakon et al. [76] introduced the second-order $(p, q)$-difference equations with separated boundary conditions:

$$
\left\{\begin{array}{l}
D_{p, q}^{2} x(t)=f\left(t, x\left(p^{2} t\right)\right), t \in\left[0, T / p^{2}\right], \\
\alpha_{1} x(0)+\alpha_{2} D_{p, q} x(0)=\alpha_{3}, \quad \beta_{1} x(T)+\beta_{2} D_{p, q} x(T / p)=\beta_{3},
\end{array}\right.
$$

where $f \in \mathcal{C}\left(\left[0, T / p^{2}\right] \times \mathbb{R}, \mathbb{R}\right)$ and $\alpha_{i}, \beta_{i}(i=1,2,3)$ are constants. $D_{p, q}^{2}$ and $D_{q}$ are the second and first order of the $(p, q)$-difference operator, respectively.

However, the study of nonlocal boundary value problems of fractional $(p, q)$-difference equations is at its infancy, and much of the work on the topic is yet to be done.

In this paper, we study the existence and uniqueness of solutions of the nonlocal boundary value problem of nonlinear fractional $(p, q)$-difference equations given by

$$
\left\{\begin{array}{l}
{ }^{c} D_{p, q}^{\alpha} y(t)=h\left(t, y\left(p^{\alpha} t\right)\right), t \in\left[0, T / p^{\alpha}\right], 1<\alpha \leq 2, \\
\beta_{1} y(0)+\gamma_{1} D_{p, q} y(0)=\zeta_{1} y\left(\eta_{1}\right), \quad \beta_{2} y(T)+\gamma_{2} D_{p, q} y(T / p)=\zeta_{2} y\left(\eta_{2}\right),
\end{array}\right.
$$

where $h \in \mathcal{C}\left(\left[0, T / p^{\alpha}\right] \times \mathbb{R}, \mathbb{R}\right)$ and $\beta_{i}, \gamma_{i}, \eta_{i}(i=1,2)$ are constants; ${ }^{c} D_{p, q}^{\alpha}$ and $D_{p, q}$ are the fractional $(p, q)$-derivative of the Caputo type and the first-order $(p, q)$-difference operator, respectively.

\section{Preliminaries}

In this section, we give some definitions and fundamental results of the $q$-calculus and $(p, q)$-calculus along with the fractional $(p, q)$-calculus, which can be found in $[27,28,56,58]$. We also give a lemma that will be used in obtaining the main results of the paper.

Throughout this paper, let $[a, b] \subset \mathbb{R}$ be an interval with $a<b$, and $0<q<p \leq 1$ be constants, 


$$
\begin{aligned}
{[k]_{p, q} } & =\frac{p^{k}-q^{k}}{p-q}, \quad k \in \mathbb{N}, \\
{[k]_{p, q} ! } & = \begin{cases}{[k]_{p, q}[k-1]_{p, q} \cdots[1]_{p, q}=\prod_{i=1}^{k} \frac{p^{i}-q^{i}}{p-q},} & k \in \mathbb{N}, \\
1, & k=0 .\end{cases}
\end{aligned}
$$

The $q$-analogue of the power function $(a-b)_{q}^{(n)}$ with $n \in \mathbb{N}_{0}:=\{0,1,2, \ldots\}$ is given by

$$
(a-b)_{q}^{(0)}=1, \quad(a-b)_{q}^{(n)}:=\prod_{k=0}^{n-1}\left(a-b q^{k}\right), \quad a, b \in \mathbb{R} .
$$

The $(p, q)$-analogue of the power function $(a-b)_{p, q}^{(n)}$ with $n \in \mathbb{N}_{0}:=\{0,1,2, \ldots\}$ is given by

$$
(a-b)_{p, q}^{(0)}=1, \quad(a-b)_{p, q}^{(n)}:=\prod_{k=0}^{n-1}\left(a p^{k}-b q^{k}\right), \quad a, b \in \mathbb{R} .
$$

For $t \in \mathbb{R} \backslash\{0,-1,-2, \ldots\}$, the $(p, q)$-gamma function is defined by

$$
\Gamma_{p, q}(t)=\frac{(p-q)_{p, q}^{(t-1)}}{(p-q)^{t-1}}
$$

and an equivalent definition of (7) is given in [59] as

$$
\Gamma_{p, q}(t)=p^{\frac{t(t-1)}{2}} \int_{0}^{\infty} x^{t-1} E_{p, q}^{-q x} d_{p, q} x,
$$

where

$$
E_{p, q}^{-q x}=\sum_{n=0}^{\infty} \frac{q^{\left(\begin{array}{c}
n \\
2
\end{array}\right)}}{[n]_{p, q} !}(-q x)^{n} .
$$

Obviously, $\Gamma_{p, q}(t+1)=[t]_{p, q} \Gamma_{p, q}(t)$.

For $s, t>0$, the definition of the $(p, q)$-beta function is

$$
B_{p, q}(s, t)=\int_{0}^{1} u^{s-1}(1-q u)_{p, q}^{(t-1)} d_{p, q} u,
$$

and (9) can also be written as

$$
B_{p, q}(s, t)=p^{(t-1)(2 s+t-2) / 2} \frac{\Gamma_{p, q}(s) \Gamma_{p, q}(t)}{\Gamma_{p, q}(s+t)},
$$

see $[77,78]$ for more details.

Definition 1 ([56]). If $f:[0, T] \rightarrow \mathbb{R}$ is a continuous function, then the $(p, q)$-derivative of $f$ is defined by

$$
D_{p, q} f(t)=\frac{f(p t)-f(q t)}{(p-q) t}, t \neq 0
$$

and $D_{p, q} f(0)=\lim _{t \rightarrow 0} D_{p, q} f(t)$. Observe that the function $D_{p, q} f(t)$ is defined on $[0, T / p]$ provided that $D_{p, q} f(t)$ exists for all $t \in[0, T / p]$.

Definition 2 ([56]). If $f:[0, T] \rightarrow \mathbb{R}$ is a continuous function, then the $(p, q)$-integral of $f$ is defined as 


$$
\int_{0}^{t} f(s) d_{p, q} s=(p-q) t \sum_{n=0}^{\infty} \frac{q^{n}}{p^{n+1}} f\left(\frac{q^{n}}{p^{n+1}} t\right)
$$

provided that the right-hand side converges. Note that the function $\int_{0}^{t} f(s) d_{p, q} s$ is defined on $[0, p T]$, which is extended from $[0, T]$ of a function $f(t)$.

Theorem 1 ([56]). Let $f, g:[0, T] \rightarrow \mathbb{R}, g(t) \neq 0$ for all $t \in[0, T]$, be $(p, q)$-differentiable on $[0, T / p]$ and $\lambda$ be a constant. Then,

(i) $D_{p, q}(f(t)+g(t))=D_{p, q} f(t)+D_{p, q} g(t)$;

(ii) $D_{p, q} \lambda f(s)=\lambda D_{p, q} f(s)$;

(iii) $D_{p, q}(f g)(t)=f(p t) D_{p, q} g(t)+g(q t) D_{p, q} f(t)$;

(iv) $D_{p, q}(f / g)(t)=\frac{g(q t) D_{p, q} f(t)-f(q t) D_{p, q} g(t)}{g(p t) g(q t)}$.

Theorem 2 ([79]). Let $f:[0, T] \rightarrow \mathbb{R}$ be a continuous function. The following formulas hold for $t \in[a, b]:$

(i) $D_{p, q} \int_{0}^{t} f(s) d_{p, q} s=f(t)$;
(ii) $\int_{0}^{t} D_{p, q} f(s) d_{p, q} s=f(t)-f(0)$;
(iii) $\int_{c}^{t} D_{p, q} f(s) d_{p, q} s=f(t)-f(c)$, for $c \in(0, t)$.

Definition 3 ([77]). Let $f:[0, T] \rightarrow \mathbb{R}$ be a continuous function and $\alpha \geq 0$. The fractional $(p, q)$-integral of fractional Riemann-Liouville type is given by $\left(I_{p, q}^{0} f\right)(t)=f(t)$ and

$$
\begin{aligned}
\left(I_{p, q}^{\alpha} f\right)(t) & =\frac{1}{p^{\left(\begin{array}{l}
\alpha \\
2
\end{array}\right)} \Gamma_{p, q}(\alpha)} \int_{0}^{t}(t-q s)_{p, q}^{(\alpha-1)} f\left(\frac{s}{p^{\alpha-1}}\right) d_{p, q} s \\
& =\frac{(p-q) t}{p^{\left(\frac{\alpha}{2}\right)} \Gamma_{p, q}(\alpha)} \sum_{n=0}^{\infty} \frac{q^{n}}{p^{n+1}}\left(t-\frac{q^{n+1}}{p^{n+1}} t\right)_{p, q}^{(\alpha-1)} f\left(\frac{q^{n}}{p^{\alpha+n}} t\right),
\end{aligned}
$$

where $t \in\left[0, p^{\alpha} T\right]$.

Definition 4 ([77]). The fractional $(p, q)$-derivative of Riemann-Liouville type of order $\alpha \geq 0$ of a continuous function $f$ is defined by $\left(D_{p, q}^{0} f\right)(t)=f(t)$ and

$$
\left(D_{p, q}^{\alpha} f\right)(t)=\left(D_{p, q}^{[\alpha]} I_{p, q}^{[\alpha]-\alpha} f\right)(t)
$$

where $[\alpha]$ is the smallest integer greater than or equal to $\alpha$.

Definition 5 ([77]). The fractional $(p, q)$-derivative of Caputo type of order $\alpha \geq 0$ of a continuous function $f$ is defined by $\left({ }^{c} D_{p, q}^{0} f\right)(t)=f(t)$ and

$$
\left({ }^{c} D_{p, q}^{\alpha} f\right)(t)=\left(I_{p, q}^{[\alpha]-\alpha} D_{p, q}^{[\alpha]} f\right)(t),
$$

where $[\alpha]$ is the smallest integer greater than or equal to $\alpha$.

Lemma 1 ([77]). Let $f$ be a continuous function and $\alpha, \beta \geq 0$. Then, the following formulas hold:

(i) $\left(I_{p, q}^{\beta} I_{p, q}^{\alpha} f\right)(t)=\left(I_{p, q}^{\alpha+\beta} f\right)(t)$,

(ii) $\left(D_{p, q}^{\alpha} I_{p, q}^{\alpha} f\right)(t)=f(t)$. 
Lemma 2 ([77]). Let $f$ be a continuous function, $\alpha \geq 0$, and $n \in \mathbb{N}$. Then, the following equality holds:

$$
\left(I_{p, q}^{\alpha} D_{p, q}^{n} f\right)(t)=\left(D_{p, q}^{n} I_{p, q}^{\alpha} f\right)(t)-\sum_{k=0}^{[\alpha]-1} \frac{t^{\alpha-n+k}}{p^{(\alpha)} \Gamma_{p, q}(\alpha-n+k+1)}\left(D_{p, q}^{k} f\right)(0) .
$$

Lemma 3 ([77]). Let $f$ be a continuous function and $\alpha \geq 0$. Then, the following equality holds:

$$
\left(I_{p, q}^{\alpha}{ }^{c} D_{p, q}^{\alpha} f\right)(t)=f(t)-\sum_{k=0}^{[\alpha]-1} \frac{t^{k}}{p^{\left(\begin{array}{c}
\alpha \\
2
\end{array}\right)} \Gamma_{p, q}(k+1)}\left(D_{p, q}^{k} f\right)(0) .
$$

In order to define the solution of the boundary value problem (4), we need the following lemma.

Lemma 4. Let $\alpha_{i}, \beta_{i}, \gamma_{i}(i=1,2)$ be constants and ${ }^{c} D_{p, q}^{\alpha}$ and $D_{p, q}$ be the fractional $(p, q)$ derivative of the Caputo type and the first-order $(p, q)$-difference operator, respectively. For a given $h \in \mathcal{C}\left(\left[0, T / p^{\alpha}\right], \mathbb{R}\right)$, a unique solution of the boundary value problem

$$
\left\{\begin{array}{l}
{ }^{c} D_{p, q}^{\alpha} y(t)=h(t), t \in\left[0, T / p^{\alpha}\right] \\
\beta_{1} y(0)+\gamma_{1} D_{p, q} y(0)=\zeta_{1} y\left(\eta_{1}\right) \\
\beta_{2} y(T)+\gamma_{2} D_{p, q} y(T / p)=\zeta_{2} y\left(\eta_{2}\right)
\end{array}\right.
$$

is given by

$$
\begin{aligned}
y(t)= & \int_{0}^{t} \frac{(t-q s)_{p, q}^{(\alpha-1)}}{p^{\left(\begin{array}{l}
\alpha \\
2
\end{array}\right)} \Gamma_{p, q}(\alpha)} h\left(\frac{s}{p^{\alpha-1}}\right) d_{p, q} s \\
& +\frac{\zeta_{1}}{\Delta}\left[\left(\beta_{2}-\zeta_{2}\right) t-\left(\beta_{2} T+\gamma_{2}-\zeta_{2} \eta_{2}\right)\right] \int_{0}^{\eta_{1}} \frac{\left(\eta_{1}-q s\right)_{p, q}^{(\alpha-1)}}{p^{(\alpha)} \Gamma_{p, q}(\alpha)} h\left(\frac{s}{p^{\alpha-1}}\right) d_{p, q} s \\
& +\frac{\zeta_{2}}{\Delta}\left[\left(\gamma_{1}-\zeta_{1} \eta_{1}\right)-\left(\beta_{1}-\zeta_{1}\right) t\right] \int_{0}^{\eta_{2}} \frac{\left(\eta_{2}-q s\right)_{p, q}^{(\alpha-1)}}{p^{(\alpha)} \Gamma_{p, q}(\alpha-1)} h\left(\frac{s}{p^{\alpha-1}}\right) d_{p, q} s \\
& +\frac{\beta_{2}}{\Delta}\left[\left(\beta_{1}-\zeta_{1}\right) t-\left(\gamma_{1}-\zeta_{1} \eta_{1}\right)\right] \int_{0}^{T} \frac{(T-q s)_{p, q}^{(\alpha-1)}}{p^{(\alpha)} \Gamma_{p, q}(\alpha-1)} h\left(\frac{s}{p^{\alpha-1}}\right) d_{p, q} s \\
& +\frac{\gamma_{2}}{\Delta}\left[\left(\gamma_{1}-\zeta_{1}\right) t-\left(\gamma_{1}-\zeta_{1} \eta_{1}\right)\right] \int_{0}^{T / p} \frac{(T / p-q s)_{p, q}^{(\alpha-2)}}{p^{\left(\frac{\alpha}{2}\right)} \Gamma_{p, q}(\alpha-1)} h\left(\frac{s}{p^{\alpha-2}}\right) d_{p, q} s,
\end{aligned}
$$

where

$$
\Delta=\left(\beta_{2}-\gamma_{2}\right)\left(\gamma_{1}-\zeta_{1} \eta_{2}\right)-\left(\beta_{1}-\zeta_{1}\right)\left(\beta_{2} T+\gamma_{2}-\zeta_{2} \eta_{2}\right) \neq 0 .
$$

Proof. By taking the fractional $(p, q)$-integral on (16) and applying Lemma 3, we have

$$
y(t)=\int_{0}^{t} \frac{(t-q s)^{(\alpha-1)}}{p^{(\alpha)} \Gamma_{p, q}(\alpha)} h\left(\frac{s}{p^{\alpha-1}}\right) d_{p, q} s+t c_{0}+c_{1}
$$

where $c_{0}, c_{1}$ are constants and $t \in[0, T]$.

Applying the above boundary condition of (16), we obtain 


$$
\left\{\begin{array}{l}
\left(\gamma_{1}-\zeta_{1} \eta_{1}\right) c_{1}+\left(\beta_{1}-\zeta_{1}\right) c_{2}=\zeta_{1} \int_{0}^{\eta_{1}} \frac{\left(\eta_{1}-q s\right)_{p, q}^{(\alpha-1)}}{p^{\left(\frac{\alpha}{2}\right)} \Gamma_{p, q}(\alpha)} h\left(\frac{s}{p^{\alpha-1}}\right) d_{p, q} s, \\
\left(\beta_{2} T+\gamma_{2}-\zeta_{2} \eta_{2}\right) c_{1}+\left(\beta_{2}-\zeta_{2}\right) c_{2}=\zeta_{2} \int_{0}^{\eta_{2}} \frac{\left(\eta_{2}-q s\right)_{p, q}^{(\alpha-1)}}{p^{\left(\frac{\alpha}{2}\right)} \Gamma_{p, q}(\alpha-1)} h\left(\frac{s}{p^{\alpha-1}}\right) d_{p, q} s \\
\quad-\beta_{2} \int_{0}^{T} \frac{(T-q s)^{(\alpha-1)}}{p^{(\alpha)} \Gamma_{p, q}(\alpha)} h\left(\frac{s}{p^{\alpha-1}}\right) d_{p, q} s \\
-\gamma_{2} \int_{0}^{T / p} \frac{(T / p-q s)^{(\alpha-2)}}{p^{(\alpha-1)} \Gamma_{p, q}(\alpha-1)} h\left(\frac{s}{p^{\alpha-2}}\right) d_{p, q} s .
\end{array}\right.
$$

Solving the above system of (19) to find the constants $c_{1}, c_{2}$, we have

$$
\begin{aligned}
c_{1}= & \frac{1}{\Delta}\left[\zeta_{1}\left(\beta_{2}-\zeta_{2}\right) \int_{0}^{\eta_{1}} \frac{\left(\eta_{1}-q s\right)_{p, q}^{(\alpha-1)}}{p^{(\alpha)} \Gamma_{p, q}(\alpha)} h\left(\frac{s}{p^{\alpha-1}}\right) d_{p, q} s\right. \\
& -\zeta_{2}\left(\beta_{1}-\zeta_{1}\right) \int_{0}^{\eta_{2}} \frac{\left(\eta_{2}-q s\right)_{p, q}^{(\alpha-1)}}{p^{\left(\begin{array}{c}
\alpha \\
2
\end{array}\right)} \Gamma_{p, q}(\alpha-1)} h\left(\frac{s}{p^{\alpha-1}}\right) d_{p, q} s \\
& +\beta_{2}\left(\beta_{1}-\zeta_{1}\right) \int_{0}^{T} \frac{(T-q s)^{(\alpha-1)}}{p^{\left(\frac{\alpha}{2}\right)} \Gamma_{p, q}(\alpha)} h\left(\frac{s}{p^{\alpha-1}}\right) d_{p, q} s \\
& \left.+\gamma_{2}\left(\beta_{1}-\zeta_{1}\right) \int_{0}^{T / p} \frac{(T / p-q s)^{(\alpha-2)}}{p^{\left(\frac{\alpha}{2}\right)} \Gamma_{p, q}(\alpha)} h\left(\frac{s}{p^{\alpha-2}}\right) d_{p, q} s\right],
\end{aligned}
$$

and

$$
\begin{aligned}
c_{2}= & \frac{1}{\Delta}\left[-\zeta_{1}\left(\beta_{2} T+\gamma_{2}-\zeta_{2} \eta_{2}\right) \int_{0}^{\eta_{1}} \frac{\left(\eta_{1}-q s\right)_{p, q}^{(\alpha-1)}}{p^{(\alpha)} \Gamma_{p, q}(\alpha)} h\left(\frac{s}{p^{\alpha-1}}\right) d_{p, q} s\right. \\
& +\zeta_{2}\left(\gamma_{1}-\zeta_{1} \eta_{1}\right) \int_{0}^{T} \frac{(T-q s)^{(\alpha-1)}}{p^{\left(\begin{array}{c}
\alpha \\
2
\end{array}\right)} \Gamma_{p, q}(\alpha)} h\left(\frac{s}{p^{\alpha-1}}\right) d_{p, q} s \\
& \left.-\beta_{1} \beta_{2} \int_{0}^{T / p} \frac{(T / p-q s)^{(\alpha-2)}}{p^{\left(\frac{(\alpha-1}{2}\right)} \Gamma_{p, q}(\alpha-1)} h\left(\frac{s}{p^{\alpha-2}}\right) d_{p, q} s\right] .
\end{aligned}
$$

Substituting the values of $c_{0}, c_{1}$ in (18), we obtain (17). We can prove the converse by direct computation. Therefore, the proof is completed.

\section{Main Results}

In view of Lemma 4 , we define an operator $\Psi: \mathcal{C}([0, T], \mathbb{R}) \rightarrow \mathcal{C}([0, T], \mathbb{R})$ as

$$
\begin{aligned}
(\Psi y)(t)= & \int_{0}^{t} \frac{(t-q s)_{p, q}^{(\alpha-1)}}{p^{(\alpha)} \Gamma_{p, q}(\alpha)} h\left(s, y\left(p^{\alpha-1} s\right)\right) d_{p, q} s \\
& +\frac{\zeta_{1}}{\Delta}\left[\left(\beta_{2}-\zeta_{2}\right) t-\left(\beta_{2} T+\gamma_{2}-\zeta_{2} \eta_{2}\right)\right] \int_{0}^{\eta_{1}} \frac{\left(\eta_{1}-q s\right)_{p, q}^{(\alpha-1)}}{p^{(\alpha)} \Gamma_{p, q}(\alpha)} h\left(s, y\left(p^{\alpha-1} s\right)\right) d_{p, q} s \\
& +\frac{\zeta_{2}}{\Delta}\left[\left(\gamma_{1}-\zeta_{1} \eta_{1}\right)-\left(\beta_{1}-\zeta_{1}\right) t\right] \int_{0}^{\eta_{2}} \frac{\left(\eta_{2}-q s\right)_{p, q}^{(\alpha-1)}}{p^{(\alpha)} \Gamma_{p, q}(\alpha-1)} h\left(s, y\left(p^{\alpha-1} s\right)\right) d_{p, q} s \\
& +\frac{\beta_{2}}{\Delta}\left[\left(\beta_{1}-\zeta_{1}\right) t-\left(\gamma_{1}-\zeta_{1} \eta_{1}\right)\right] \int_{0}^{T} \frac{(T-q s)_{p, q}^{(\alpha-1)}}{p^{\left(\begin{array}{c}
\alpha \\
2
\end{array}\right)} \Gamma_{p, q}(\alpha-1)} h\left(s, y\left(p^{\alpha-1} s\right)\right) d_{p, q} s
\end{aligned}
$$




$$
+\frac{\gamma_{2}}{\Delta}\left[\left(\beta_{1}-\zeta_{1}\right) t-\left(\gamma_{1}-\zeta_{1} \eta_{1}\right)\right] \int_{0}^{T / p} \frac{(T / p-q s)_{p, q}^{(\alpha-2)}}{p^{(\alpha-1} \Gamma_{p, q}(\alpha-1)} h\left(s, y\left(p^{\alpha-2} s\right)\right) d_{p, q} s,
$$

where

$$
\Delta=\left(\beta_{2}-\zeta_{2}\right)\left(\gamma_{1}-\zeta_{1} \eta_{2}\right)-\left(\beta_{1}-\zeta_{1}\right)\left(\beta_{2} T+\gamma_{2}-\zeta_{2} \eta_{2}\right) \neq 0 .
$$

Let $\Omega:=\mathcal{C}([0, T], \mathbb{R})$ denote the Banach space of all continuous functions from $[0, T]$ to $\mathbb{R}$ endowed with norm defined by $\|y\|=\max \{|y(t)|: t \in[0, T]\}$.

Observe that the boundary value problem (4) has a unique solution if the operator equation $\Psi y=y$ has a fixed point, where $\Psi$ is given in (20). For convenience, we let

$$
\begin{aligned}
k= & \left(I_{p, q}^{\alpha} \lambda\right)(T)+\left|\zeta_{1}\right| \sigma_{1}(T)\left(I_{p, q}^{\alpha} \lambda\right)\left(\eta_{1}\right)+\left|\zeta_{2}\right| \sigma_{2}(T)\left(I_{p, q}^{\alpha} \lambda\right)\left(\eta_{2}\right) \\
& +\left|\beta_{2}\right| \sigma_{2}(T)\left(I_{p, q}^{\alpha} \lambda\right)(T)+\left|\gamma_{2}\right| \sigma_{2}(T)\left(I_{p, q}^{\alpha-1} \lambda\right)(T / p),
\end{aligned}
$$

where

$$
\sigma_{1}(T)=\frac{\left(\left|\beta_{2}-\zeta_{2}\right| T+\left|\beta_{2} T+\gamma_{2}-\zeta_{2} \eta_{2}\right|\right)}{|\Delta|}
$$

and

$$
\sigma_{2}(T)=\frac{\left(\left|\beta_{1}-\zeta_{1}\right| T+\left|\gamma_{1}-\zeta_{1} \eta_{1}\right|\right)}{|\Delta|} .
$$

Theorem 3. Let $h:\left[0, T / p^{\alpha}\right] \times \mathbb{R} \rightarrow \mathbb{R}$ be a continuous function and note that there exists $a$ $(p, q)$-integrable function $\lambda:\left[0, T / p^{\alpha}\right] \rightarrow \mathbb{R}$ such that

$\left(A_{1}\right)|h(t, y)-h(t, z)| \leq \lambda(t)|y-z|$, for each $t \in\left[0, T / p^{\alpha}\right]$ and $y, z \in \mathbb{R}$.

If

$$
k<1 \text {, }
$$

where $k$ is given by (21), then the boundary value problem (4) has a unique solution.

Proof. Let us fix $\sup _{t \in\left[0, T / p^{\alpha}\right]}|h(t, 0)|=M$ and choose

$$
r \geq \frac{M A}{1-k}
$$

where

$$
\begin{aligned}
A= & \frac{T^{\alpha}}{\Gamma_{p, q}(\alpha+1)}+\left|\beta_{2}\right| \sigma_{2}(T) \frac{T^{\alpha}}{\Gamma_{p, q}(\alpha+1)}+\left|\gamma_{2}\right| \sigma_{2}(T) \frac{T^{\alpha-1}}{\Gamma_{p, q}(\alpha)} \\
& +\left|\zeta_{1}\right| \sigma_{1}(T) \frac{\eta_{1}^{\alpha}}{\Gamma_{p, q}(\alpha+1)}+\left|\zeta_{2}\right| \sigma_{2}(T) \frac{\eta_{2}^{\alpha}}{\Gamma_{p, q}(\alpha+1)} .
\end{aligned}
$$

We define $B_{r}=\{y \in \mathbb{C}:\|y\| \leq r\}$ and we shall show that $\Psi B_{r} \subset B_{r}$, where $\Psi$ is defined (20). For any $y \in B_{r}$, observe that

$$
|h(t, y(t))| \leq|h(t, y(t))-h(t, 0)|+|h(t, 0)| \leq \lambda(t) r+M
$$

and

$$
\begin{aligned}
|(\Psi y)(t)| \leq & \int_{0}^{t} \frac{(t-q s)_{p, q}^{(\alpha-1)}}{p^{(\alpha)} \Gamma_{p, q}^{(\alpha)}}\left|h\left(s, y\left(p^{\alpha-1} s\right)\right)\right| d_{p, q} s \\
& +\left|\zeta_{1}\right| \sigma_{1}(T) \int_{0}^{\eta_{1}} \frac{\left(\eta_{1}-q s\right)_{p, q}^{(\alpha-1)}}{p^{\left(\begin{array}{c}
\alpha \\
2
\end{array}\right)} \Gamma_{p, q}(\alpha)}\left|h\left(s, y\left(p^{\alpha-1} s\right)\right)\right| d_{p, q} s
\end{aligned}
$$




$$
\begin{aligned}
& +\left|\zeta_{2}\right| \sigma_{2}(T) \int_{0}^{\eta_{2}} \frac{\left(\eta_{2}-q s\right)_{p, q}^{(\alpha-1)}}{p^{\left(\begin{array}{c}
\alpha \\
2
\end{array}\right)} \Gamma_{p, q}(\alpha-1)}\left|h\left(s, y\left(p^{\alpha-1} s\right)\right)\right| d_{p, q} s \\
& +\left|\beta_{2}\right| \sigma_{2}(T) \int_{0}^{T} \frac{(T-q s)_{p, q}^{(\alpha-1)}}{p^{\left(\frac{\alpha}{2}\right)} \Gamma_{p, q}(\alpha-1)}\left|h\left(s, y\left(p^{\alpha-1} s\right)\right)\right| d_{p, q} s \\
& +\left|\gamma_{2}\right| \sigma_{2}(T) \int_{0}^{T / p} \frac{(T / p-q s)_{p, q}^{(\alpha-2)}}{p^{\left(\begin{array}{c}
\alpha-1 \\
2
\end{array}\right)} \Gamma_{p, q}(\alpha-1)}\left|h\left(s, y\left(p^{\alpha-2} s\right)\right)\right| d_{p, q} S \\
& \leq \int_{0}^{T} \frac{(T-q s)_{p, q}^{(\alpha-1)}}{p^{\left(\begin{array}{c}
\alpha \\
2
\end{array}\right)} \Gamma_{p, q}(\alpha)}\left|\lambda\left(\frac{s}{p^{\alpha-1}}\right) r+M\right| d_{p, q} s \\
& +\left|\zeta_{1}\right| \sigma_{1}(T) \int_{0}^{\eta_{1}} \frac{\left(\eta_{1}-q s\right)_{p, q}^{(\alpha-1)}}{p^{\left(\begin{array}{c}
\alpha \\
2
\end{array}\right)} \Gamma_{p, q}(\alpha)}\left|\lambda\left(\frac{s}{p^{\alpha-1}}\right) r+M\right| d_{p, q} s \\
& +\left|\zeta_{2}\right| \sigma_{2}(T) \int_{0}^{\eta_{2}} \frac{\left(\eta_{2}-q s\right)_{p, q}^{(\alpha-1)}}{p^{\left(\begin{array}{c}
\alpha \\
2
\end{array}\right)} \Gamma_{p, q}(\alpha-1)}\left|\lambda\left(\frac{s}{p^{\alpha-1}}\right) r+M\right| d_{p, q} s \\
& +\left|\beta_{2}\right| \sigma_{2}(T) \int_{0}^{T} \frac{(T-q s)_{p, q}^{(\alpha-1)}}{p^{\left(\begin{array}{c}
\alpha \\
2
\end{array}\right)} \Gamma_{p, q}(\alpha-1)}\left|\lambda\left(\frac{s}{p^{\alpha-2}}\right) r+M\right| d_{p, q} S \\
& +\left|\gamma_{2}\right| \sigma_{2}(T) \int_{0}^{T / p} \frac{(T / p-q s)_{p, q}^{(\alpha-2)}}{p^{\left(\frac{\alpha-1}{2}\right)} \Gamma_{p, q}(\alpha-1)}\left|\lambda\left(\frac{s}{p^{\alpha-2}}\right) r+M\right| d_{p, q} S \\
& \leq M\left\{\frac{T^{\alpha}}{\Gamma_{p, q}(\alpha+1)}+\left|\beta_{2}\right| \sigma_{1}(T) \frac{T^{\alpha}}{\Gamma_{p, q}(\alpha+1)}+\left|\gamma_{2}\right| \sigma_{2}(T) \frac{T^{\alpha-1}}{\Gamma_{p, q}(\alpha)}\right. \\
& \left.+\left|\zeta_{1}\right| \sigma_{2}(T) \frac{\eta_{1}^{\alpha}}{\Gamma_{p, q}(\alpha+1)}+\left|\zeta_{2}\right| \sigma_{2}(T) \frac{\eta_{1}^{\alpha}}{\Gamma_{p, q}(\alpha+1)}\right\} \\
& +r\left\{\int_{0}^{T} \frac{(T-q s)^{(\alpha-1)}}{p^{\left(\begin{array}{c}
\alpha \\
2
\end{array}\right)} \Gamma_{p, q}(\alpha)} \lambda\left(\frac{s}{p^{\alpha-1}}\right) d_{p, q} S\right. \\
& +\left|\zeta_{1}\right| \sigma_{1}(T) \int_{0}^{\eta_{1}} \frac{\left(\eta_{1}-q s\right)_{p, q}^{(\alpha-1)}}{p^{\left(\begin{array}{c}
\alpha \\
2
\end{array}\right)} \Gamma_{p, q}(\alpha)} \lambda\left(\frac{s}{p^{\alpha-1}}\right) d_{p, q} S \\
& +\left|\zeta_{2}\right| \sigma_{2}(T) \int_{0}^{\eta_{2}} \frac{\left(\eta_{2}-q s\right)_{p, q}^{(\alpha-1)}}{p^{\left(\frac{\alpha}{2}\right)} \Gamma_{p, q}(\alpha)} \lambda\left(\frac{s}{p^{\alpha-1}}\right) d_{p, q} s \\
& +\left|\beta_{2}\right| \sigma_{2}(T) \int_{0}^{T} \frac{(T-q s)^{(\alpha-1)}}{p^{(\alpha)} \Gamma_{p, q}^{(\alpha)}(\alpha)} \lambda\left(\frac{s}{p^{\alpha-1}}\right) d_{p, q} S \\
& \left.+\left|\gamma_{2}\right| \sigma_{2}(T) \int_{0}^{T / p} \frac{(T / p-q s)_{p, q}^{(\alpha-2)}}{p^{(\alpha-1)} \Gamma_{p, q}(\alpha-1)} \lambda\left(\frac{s}{p^{\alpha-2}}\right) d_{p, q} s\right\},
\end{aligned}
$$

which, in view of (21) and (23), implies that

$$
\|\Psi y\| \leq M A+r k \leq r .
$$

This implies that $\Psi y \subset B_{r}$.

Now, for $y, z \in \mathcal{C}$, we obtain 


$$
\begin{aligned}
& \|\Psi y-\Psi z\| \\
& \leq \sup _{t \in\left[0, T / p^{\alpha}\right]}\left\{\int_{0}^{t} \frac{(t-q s)_{p, q}^{(\alpha-1)}}{p^{\left(\begin{array}{c}
\alpha \\
2
\end{array}\right)} \Gamma_{p, q}(\alpha)}\left|h\left(s, y\left(p^{\alpha-1} s\right)\right)-h\left(s, z\left(p^{\alpha-1} s\right)\right)\right| d_{p, q} s\right. \\
& +\left|\zeta_{1}\right| \sigma_{1}(T) \int_{0}^{\eta_{1}} \frac{\left(\eta_{1}-q s\right)_{p, q}^{(\alpha-1)}}{p^{\left(\begin{array}{c}
\alpha \\
2
\end{array}\right)} \Gamma_{p, q}(\alpha)}\left|h\left(s, y\left(p^{\alpha-1} s\right)\right)-h\left(s, z\left(p^{\alpha-1} s\right)\right)\right| d_{p, q} s \\
& +\left|\zeta_{2}\right| \sigma_{2}(T) \int_{0}^{\eta_{2}} \frac{\left(\eta_{2}-q s\right)_{p, q}^{(\alpha-1)}}{p^{\left(\begin{array}{c}
\alpha \\
2
\end{array}\right)} \Gamma_{p, q}(\alpha)}\left|h\left(s, y\left(p^{\alpha-1} s\right)\right)-h\left(s, z\left(p^{\alpha-1} s\right)\right)\right| d_{p, q} s \\
& +\left|\beta_{2}\right| \sigma_{2}(T) \int_{0}^{T} \frac{(T-q s)_{p, q}^{(\alpha-1)}}{p^{\left(\begin{array}{c}
\alpha \\
2
\end{array}\right)} \Gamma_{p, q}(\alpha)}\left|h\left(s, y\left(p^{\alpha-1} s\right)\right)-h\left(s, z\left(p^{\alpha-1} s\right)\right)\right| d_{p, q} s \\
& \left.+\left|\gamma_{2}\right| \sigma_{2}(T) \int_{0}^{T / p} \frac{(T / p-q s)_{p, q}^{(\alpha-2)}}{p^{(\alpha)} \Gamma_{p, q}(\alpha)}\left|h\left(s, y\left(p^{\alpha} s\right)\right)-h\left(s, z\left(p^{\alpha} s\right)\right)\right| d_{p, q} s\right\} \\
& \leq\|y-z\|\left\{\int_{0}^{T} \frac{(T-q s)_{p, q}^{(\alpha-1)}}{p^{\left(\begin{array}{c}
\alpha \\
2
\end{array}\right)} \Gamma_{p, q}(\alpha)} \lambda\left(\frac{s}{p^{\alpha-1}}\right) d_{p, q} S\right. \\
& +\left|\zeta_{1}\right| \sigma_{1}(T) \int_{0}^{\eta_{1}} \frac{\left(\eta_{1}-q s\right)_{p, q}^{(\alpha-1)}}{p^{\left(\begin{array}{c}
\alpha \\
2
\end{array}\right)} \Gamma_{p, q}(\alpha)} \lambda\left(\frac{s}{p^{\alpha-1}}\right) d_{p, q} S \\
& +\left|\zeta_{2}\right| \sigma_{2}(T) \int_{0}^{\eta_{2}} \frac{\left(\eta_{2}-q s\right)_{p, q}^{(\alpha-1)}}{p^{\left(\begin{array}{c}
\alpha \\
2
\end{array}\right)} \Gamma_{p, q}(\alpha)} \lambda\left(\frac{s}{p^{\alpha-1}}\right) d_{p, q} S \\
& +\sigma_{1}(T)\left|\beta_{2}\right| \sigma_{2}(T) \int_{0}^{T} \frac{(T-q s)_{p, q}^{(\alpha-1)}}{p^{(\alpha)} \Gamma_{p, q}(\alpha)} \lambda\left(\frac{s}{p^{\alpha-1}}\right) d_{p, q} S \\
& \left.+\sigma_{2}(T)\left|\gamma_{2}\right| \sigma_{2}(T) \int_{0}^{T / p} \frac{(T / p-q s)_{p, q}^{(\alpha-2)}}{p^{\left({ }^{\alpha-1}\right)} \Gamma_{p, q}(\alpha-1)} \lambda\left(\frac{s}{p^{\alpha-2}}\right) d_{p, q} s\right\},
\end{aligned}
$$

from which, in view of (21), we obtain

$$
\|\Psi y-\Psi z\| \leq k\|y-z\|
$$

Because $k \in(0,1)$ by assumptions (22), therefore, $\Psi$ is a contraction. Thus, it follows by Banach's contraction principle that the boundary value problem (4) has a unique solution.

In case $\lambda(t)=\lambda$, where $\lambda$ is a constant, the condition (22) becomes $\lambda A<1$ and Theorem 3 takes the form of the following results.

Remark 1. If $h:\left[0, T / p^{\alpha}\right] \times \mathbb{R} \rightarrow \mathbb{R}$ is a contraction function and there exists a constant $\lambda>0$ with

$$
|h(t, y)-h(t, z)| \leq \lambda|y-z|,
$$

for each $t \in\left[0, T / p^{\alpha}\right]$ and $y, z \in \mathbb{R}$, then the boundary value problem (4) has a unique solution, provided that $k<1$.

Our next existence results are based on Krasnoselskii's fixed-point theorem.

Lemma 5 ([80]). (Krasnoselskii's fixed-point theorem) Let $M$ be a closed, bounded, convex, and non-empty subset of a Banach space X. Let $A, B$ be two operators such that:

(i) $A y+B z \in M$, whenever $y, z \in M$;

(ii) $A$ is compact and continuous; 
(iii) $B$ is a contraction mapping.

Then, there exists $x \in M$ such that $x=A x+B x$.

Theorem 4. Let $h:\left[0, T / p^{\alpha}\right] \times \mathbb{R} \rightarrow \mathbb{R}$ be a continuous function satisfying $\left(A_{1}\right)$. In addition, we assume that

$\left(A_{2}\right)$ there exists a function $\mu \in \mathcal{C}\left(\left[0, T / p^{\alpha}\right], \mathbb{R}^{+}\right)$and a non-decreasing function $\phi \in \mathcal{C}\left(\left[0, T / p^{\alpha}\right]\right.$, $\mathbb{R}^{+}$) with

$$
|h(t, y)| \leq \mu(t) \phi(|y|),
$$

where $(t, y) \in\left[0, T / p^{\alpha}\right] \times \mathbb{R}$

If

$$
\begin{aligned}
& \int_{0}^{T} \frac{(T-q s)_{p, q}^{(\alpha-1)}}{p^{(\alpha)} \Gamma_{p, q}(\alpha)} \lambda\left(\frac{s}{p^{\alpha-1}}\right) d_{p, q} s \\
& +\left|\zeta_{1}\right| \sigma_{1}(T) \int_{0}^{\eta_{1}} \frac{\left(\eta_{1}-q s\right)_{p, q}^{(\alpha-1)}}{p^{(\alpha)} \Gamma_{p, q}^{(\alpha)}(\alpha)} \lambda\left(\frac{s}{p^{\alpha-1}}\right) d_{p, q} s \\
& +\left|\zeta_{2}\right| \sigma_{2}(T) \int_{0}^{\eta_{2}} \frac{\left(\eta_{2}-q s\right)_{p, q}^{(\alpha-1)}}{p^{(\alpha)} \Gamma_{p, q}^{(\alpha)}} \lambda\left(\frac{s}{p^{\alpha-1}}\right) d_{p, q} S \\
& +\sigma_{1}(T)\left|\beta_{2}\right| \sigma_{2}(T) \int_{0}^{T} \frac{(T-q s)_{p, q}^{(\alpha-1)}}{p^{\left(\begin{array}{c}
\alpha \\
2
\end{array}\right)} \Gamma_{p, q}(\alpha)} \lambda\left(\frac{s}{p^{\alpha-1}}\right) d_{p, q} s \\
& +\sigma_{2}(T)\left|\gamma_{2}\right| \sigma_{2}(T) \int_{0}^{T / p} \frac{(T / p-q s)_{p, q}^{(\alpha-2)}}{p^{\left(\frac{\alpha-1}{2}\right)} \Gamma_{p, q}(\alpha-1)} \lambda\left(\frac{s}{p^{\alpha-2}}\right) d_{p, q} s<1,
\end{aligned}
$$

then the boundary value problem (4) has at least one solution on $[0, T]$.

Proof. Let $\mathcal{B}_{\bar{r}}:=\{y \in \mathcal{C}:\|y\| \leq \bar{r}\}$, where

$$
\begin{aligned}
\bar{r} & \geq \phi(\bar{r})\|\mu\|\left\{\frac{T^{\alpha}}{\Gamma_{p, q}(\alpha+1)}+\left|\beta_{2}\right| \sigma_{1}(T) \frac{T^{\alpha}}{\Gamma_{p, q}(\alpha+1)}+\left|\gamma_{2}\right| \sigma_{2}(T) \frac{T^{\alpha-1}}{\Gamma_{p, q}(\alpha)}\right. \\
& \left.+\left|\zeta_{1}\right| \sigma_{2}(T) \frac{\eta_{1}^{\alpha}}{\Gamma_{p, q}(\alpha+1)}+\left|\zeta_{2}\right| \sigma_{2}(T) \frac{\eta_{1}^{\alpha}}{\Gamma_{p, q}(\alpha+1)}\right\},
\end{aligned}
$$

where $\|\mu\|=\sup _{\left[0, T / p^{\alpha}\right]}|\mu(t)|$ and define the operators $\mathcal{P}$ and $\mathcal{Q}$ on $\mathcal{B}_{\bar{r}}$ as

$$
(\mathcal{P} y)(t)=\int_{0}^{t} \frac{(t-q s)^{(\alpha-1)}}{p^{(\alpha)} \Gamma_{p, q}(\alpha)} h\left(s, y\left(p^{\alpha-1} s\right)\right) d_{p, q} S
$$

and

$$
\begin{aligned}
(\mathcal{Q} y)(t)= & \frac{\zeta_{1}}{\Delta}\left[\left(\beta_{2}-\zeta_{2}\right) t-\left(\beta_{2} T+\gamma_{2}-\zeta_{2} \eta_{2}\right)\right] \int_{0}^{\eta_{1}} \frac{\left(\eta_{1}-q s\right)_{p, q}^{(\alpha-1)}}{p^{(\alpha)} \Gamma_{p, q}(\alpha)} h\left(s, y\left(p^{\alpha-1} s\right)\right) d_{p, q} s \\
& +\frac{\zeta_{2}}{\Delta}\left[\left(\gamma_{1}-\zeta_{1} \eta_{1}\right)-\left(\beta_{1}-\zeta_{1}\right) t\right] \int_{0}^{\eta_{2}} \frac{\left(\eta_{2}-q s\right)_{p, q}^{(\alpha-1)}}{p^{(\alpha)} \Gamma_{p, q}(\alpha-1)} h\left(s, y\left(p^{\alpha-1} s\right)\right) d_{p, q} s \\
& +\frac{\beta_{2}}{\Delta}\left[\left(\beta_{1}-\zeta_{1}\right) t-\left(\gamma_{1}-\zeta_{1} \eta_{1}\right)\right] \int_{0}^{T} \frac{(T-q s)_{p, q}^{(\alpha-1)}}{p^{(\alpha)} \Gamma_{p, q}(\alpha-1)} h\left(s, y\left(p^{\alpha-1} s\right)\right) d_{p, q} s \\
& +\frac{\gamma_{2}}{\Delta}\left[\left(\beta_{1}-\zeta_{1}\right) t-\left(\gamma_{1}-\zeta_{1} \eta_{1}\right)\right] \int_{0}^{T / p} \frac{(T / p-q s)_{p, q}^{(\alpha-2)}}{p^{\left(\frac{(\alpha-1}{2}\right)} \Gamma_{p, q}(\alpha-1)} h\left(s, y\left(p^{\alpha-2} s\right)\right) d_{p, q} s,
\end{aligned}
$$


where

$$
\Delta=\left(\beta_{2}-\zeta_{2}\right)\left(\gamma_{1}-\zeta_{1} \eta_{2}\right)-\left(\beta_{1}-\zeta_{1}\right)\left(\beta_{2} T+\gamma_{2}-\zeta_{2} \eta_{2}\right) \neq 0 .
$$

For $y, z \in \mathcal{B}_{\bar{r}}$, we have

$$
\begin{aligned}
& |(\mathcal{P} y+\mathcal{Q} z)(t)| \leq \int_{0}^{t} \frac{(t-q s)^{(\alpha-1)}}{p^{\left(\begin{array}{c}
\alpha \\
2
\end{array}\right)} \Gamma_{p, q}(\alpha)}\left|h\left(s, y\left(p^{\alpha-1} s\right)\right)\right| d_{p, q} s \\
& +\frac{\zeta_{1}}{\Delta}\left[\left(\beta_{2}-\zeta_{2}\right) t-\left(\beta_{2} T+\gamma_{2}-\zeta_{2} \eta_{2}\right)\right] \int_{0}^{\eta_{1}} \frac{\left(\eta_{1}-q s\right)_{p, q}^{(\alpha-1)}}{p^{\left(\begin{array}{c}
\alpha \\
2
\end{array}\right)} \Gamma_{p, q}(\alpha)} h\left(s, z\left(p^{\alpha-1} s\right)\right) d_{p, q} s \\
& +\frac{\zeta_{2}}{\Delta}\left[\left(\gamma_{1}-\zeta_{1} \eta_{1}\right)-\left(\beta_{1}-\zeta_{1}\right) t\right] \int_{0}^{\eta_{2}} \frac{\left(\eta_{2}-q s\right)_{p, q}^{(\alpha-1)}}{p^{\left(\begin{array}{c}
\alpha \\
2
\end{array}\right)} \Gamma_{p, q}(\alpha-1)} h\left(s, z\left(p^{\alpha-1} s\right)\right) d_{p, q} s \\
& +\frac{\beta_{2}}{\Delta}\left[\left(\beta_{1}-\zeta_{1}\right) t-\left(\gamma_{1}-\zeta_{1} \eta_{1}\right)\right] \int_{0}^{T} \frac{(T-q s)_{p, q}^{(\alpha-1)}}{p^{\left(\begin{array}{l}
\alpha \\
2
\end{array}\right)} \Gamma_{p, q}(\alpha-1)} h\left(s, z\left(p^{\alpha-1} s\right)\right) d_{p, q} s \\
& +\frac{\gamma_{2}}{\Delta}\left[\left(\beta_{1}-\zeta_{1}\right) t-\left(\gamma_{1}-\zeta_{1} \eta_{1}\right)\right] \int_{0}^{T / p} \frac{(T / p-q s)_{p, q}^{(\alpha-2)}}{p^{\left(\begin{array}{c}
\alpha-1 \\
2
\end{array}\right)} \Gamma_{p, q}(\alpha-1)} h\left(s, z\left(p^{\alpha-2} s\right)\right) d_{p, q} s \\
& \leq \int_{0}^{t} \frac{(t-q s)_{p, q}^{(\alpha-1)}}{p^{\left(\begin{array}{c}
\alpha \\
2
\end{array}\right)} \Gamma_{p, q}(\alpha)} \mu(t) \phi(|y|) d_{p, q} s \\
& +\left|\zeta_{1}\right| \sigma_{1}(T) \int_{0}^{\eta_{1}} \frac{\left(\eta_{1}-q s\right)_{p, q}^{(\alpha-1)}}{p^{\left(\begin{array}{c}
\alpha \\
2
\end{array}\right)} \Gamma_{p, q}(\alpha)} \mu(t) \phi(|z|) d_{p, q} s \\
& +\left|\zeta_{2}\right| \sigma_{2}(T) \int_{0}^{\eta_{2}} \frac{\left(\eta_{2}-q s\right)_{p, q}^{(\alpha-1)}}{p^{\left(\begin{array}{c}
\alpha \\
2
\end{array}\right)} \Gamma_{p, q}(\alpha-1)} \mu(t) \phi(|z|) d_{p, q} s \\
& +\left|\beta_{2}\right| \sigma_{2}(T) \int_{0}^{T} \frac{(T-q s)_{p, q}^{(\alpha-1)}}{p^{\left(\begin{array}{c}
\alpha \\
2
\end{array}\right)} \Gamma_{p, q}(\alpha-1)} \mu(t) \phi(|z|) d_{p, q} S \\
& +\left|\gamma_{2}\right| \sigma_{2}(T) \int_{0}^{T / p} \frac{(T / p-q s)_{p, q}^{(\alpha-2)}}{p^{\left(\frac{\alpha-1}{2}\right)} \Gamma_{p, q}(\alpha-1)} \mu(t) \phi(|z|) d_{p, q} s \\
& \leq \phi(\bar{r})\|\mu\|\left\{\frac{T^{\alpha}}{\Gamma_{p, q}(\alpha+1)}+\left|\beta_{2}\right| \sigma_{1}(T) \frac{T^{\alpha}}{\Gamma_{p, q}(\alpha+1)}+\left|\gamma_{2}\right| \sigma_{2}(T) \frac{T^{\alpha-1}}{\Gamma_{p, q}(\alpha)}\right. \\
& \left.+\left|\zeta_{1}\right| \sigma_{2}(T) \frac{\eta_{1}^{\alpha}}{\Gamma_{p, q}(\alpha+1)}+\left|\zeta_{2}\right| \sigma_{2}(T) \frac{\eta_{1}^{\alpha}}{\Gamma_{p, q}(\alpha+1)}\right\} \\
& \leq \bar{r} .
\end{aligned}
$$

Thus, $\mathcal{P} y+\mathcal{Q} z \in \mathcal{B}_{\bar{r}}$. From $\left(A_{1}\right)$ and (26), it follows that $\mathcal{Q}$ is a contraction mapping. By continuity of $h$, we obtain that the operator $\mathcal{P}$ is continuous. It is not difficult to verify that

$$
\|\mathcal{P}\| \leq \frac{\phi(\bar{r})\|\mu\| T^{\alpha}}{\Gamma_{p, q}(\alpha+1)} .
$$

Therefore, the set $\mathcal{P}\left(\mathcal{B}_{\bar{r}}\right)$ is uniformly bounded. Next, we shall prove the compactness of the operator $\mathcal{P}$. Now, for any $y \in B_{\bar{r}} t_{1}, t_{2} \in[0, T]$ with $t_{1}<t_{2}$. Then, we obtain 


$$
\begin{aligned}
& \left|(\mathcal{P} y)\left(t_{2}\right)-(\mathcal{Q} y)\left(t_{1}\right)\right| \\
& =\left|\int_{0}^{t_{2}} \frac{(t-q s)^{(\alpha-1)}}{p^{(\alpha)} \Gamma_{p, q}(\alpha)} f\left(s, y\left(p^{\alpha-1} s\right)\right) d_{p, q} s-\int_{0}^{t_{1}} \frac{(t-q s)^{(\alpha-1)}}{p^{(\alpha)} \Gamma_{p, q}(\alpha)} f\left(s, y\left(p^{\alpha-1} s\right)\right) d_{p, q} s\right| \\
& \leq\left|\int_{0}^{t_{1}} \frac{\left(t_{2}-q s\right)^{(\alpha-1)}-\left(t_{1}-q s\right)^{(\alpha-1)}}{p^{(\alpha)} \Gamma_{p, q}(\alpha)} f\left(s, y\left(p^{\alpha-1} s\right)\right) d_{p, q} s\right| \\
& \quad+\left|\int_{t_{1}}^{t_{2}} \frac{\left(t_{2}-q s\right)^{(\alpha-1)}}{p^{(\alpha)} \Gamma_{p, q}(\alpha)} f\left(s, y\left(p^{\alpha-1} s\right)\right) d_{p, q} s\right| \\
& \leq \phi(\bar{r})\|\mu\|\left[\int_{0}^{t_{1}} \frac{\left(t_{2}-q s\right)^{(\alpha-1)}-\left(t_{1}-q s\right)^{(\alpha-1)}}{p^{(\alpha)} \Gamma_{p, q}(\alpha)} f\left(s, y\left(p^{\alpha-1} s\right)\right) d_{p, q} s\right. \\
& \left.\quad+\int_{t_{1}}^{t_{2}} \frac{\left(t_{2}-q s\right)^{(\alpha-1)}}{p^{\left(\frac{\alpha}{2}\right)} \Gamma_{p, q}(\alpha)} f\left(s, y\left(p^{\alpha-1} s\right)\right) d_{p, q} s .\right]
\end{aligned}
$$

which is independent of $x$ and tends to zero as $t_{1} \rightarrow t_{2}$. Thus, the set $\mathcal{P}\left(B_{\bar{r}}\right)$ is equicontinuous. By the Arzelá-Ascoli theorem, $\mathcal{P}$ is compact on $B_{\bar{r}}$. Therefore, the boundary value problem (4) has at least one solution on $[0, T]$. This completes the proof.

Remark 2. Let $h:\left[0, T / p^{\alpha}\right] \times \mathbb{R} \rightarrow \mathbb{R}$ be a continuous function satisfying $\left(A_{1}\right)$. In addition, we assume that

$$
|h(t, y)| \leq \mu(t), \forall(t, y) \in\left[0, T / p^{\alpha}\right] \times \mathbb{R} \text { and } \mu \in \mathbb{C}\left(\left[0, T / p^{\alpha}\right], \mathbb{R}^{+}\right) .
$$

If (26) holds, then the boundary value problem (4) has at least one solution on $[0, T]$.

The next existence results are based on the Leray-Schauder nonlinear alternative.

Lemma 6 ([81]). (Nonlinear alternative for single value maps) Let $E$ be a Banach space, $\mathbb{C}$ a closed, convex subset of $E$, and $\mathbb{U}$ an open subset of $\mathbb{C}$ with $0 \in \mathbb{U}$. Suppose that $\Psi: \overline{\mathbb{U}} \rightarrow \mathcal{C}$ is a continuous, compact function; that is, $\Psi(\overline{\mathbb{U}})$ is a relatively compact subset of $\mathcal{C}$ map. Then, either

(i) $\Psi$ has a fixed point in $\overline{\mathbb{U}}$, or

(ii) there is a $u \in \partial \mathbb{U}$ (the boundary of $\mathbb{U}$ in $\mathbb{C}$ ) and $\lambda \in(0,1)$ with $u=\lambda \Psi(u)$.

Theorem 5. Let $h:\left[0, T / p^{\alpha}\right] \times \mathbb{R} \rightarrow \mathbb{R}$ be a continuous function. Assume that:

$\left(A_{3}\right)$ there exist functions $p_{1}, p_{2} \in \mathbb{C}\left(\left[0, T / p^{\alpha}\right], \mathbb{R}^{+}\right)$, and a non-decreasing function $\psi: \mathbb{R}^{+} \rightarrow$ $\mathbb{R}^{+}$such that

$$
|h(t, y)| \leq p_{1}(t) \psi(|y|)+p_{2}(t), t, y \in\left[0, T / p^{\alpha}\right] \times \mathbb{R} ;
$$

$\left(A_{4}\right)$ there exists a number $M>0$ such that

$$
\frac{M}{\psi(M) \omega_{1}+\omega_{2}}>1
$$

where

$$
\begin{aligned}
\omega_{i}:= & \left(1+\left|\beta_{2}\right| \sigma_{2}\right)\left(I_{p, q}^{\alpha} p_{i}\right)(T)+\left|\zeta_{1}\right| \sigma_{1}(T)\left(I_{p, q}^{\alpha} p_{i}\right)\left(\eta_{1}\right)+\left|\zeta_{2}\right| \sigma_{2}(T)\left(I_{p, q}^{\alpha} p_{i}\right)\left(\eta_{2}\right) \\
& +\left|\gamma_{2}\right| \sigma_{2}(T)\left(I_{p, q}^{\alpha-1} p_{i}\right)(T / p), i=1,2 .
\end{aligned}
$$

Then, the boundary value problem (4) has at least one solution on $[0, T]$. 
Proof. Consider the operator $\Psi: \mathcal{C} \rightarrow \mathcal{C}$ defined by (20). We first show that $\Psi$ is continuous. Let $\left\{y_{n}\right\}$ be a sequence of function such that $y_{n} \rightarrow y$ on $\left[0, T / p^{\alpha}\right]$. Given that $h$ is a continuous function on $\left[0, T / p^{\alpha}\right]$, we have

$$
h\left(t, y_{n}\left(p^{\alpha} t\right)\right) \rightarrow h\left(t, y\left(p^{\alpha} t\right)\right) .
$$

Therefore, we obtain

$$
\begin{aligned}
\left|\left(\Psi y_{n}\right)(t)-(\Psi y)(t)\right| \leq & \int_{0}^{t} \frac{(t-q s)_{p, q}^{(\alpha-1)}}{p^{\left(\begin{array}{c}
\alpha \\
2
\end{array}\right)} \Gamma_{p, q}(\alpha)}\left|h\left(s, y_{n}\left(p^{\alpha} s\right)\right)-h\left(s, y\left(p^{\alpha} s\right)\right)\right| d_{p, q} s \\
& +\left|\zeta_{1}\right| \sigma_{1}(T) \int_{0}^{\eta_{1}} \frac{\left(\eta_{1}-q s\right)_{p, q}^{(\alpha-1)}}{p^{(\alpha)} \Gamma_{p, q}(\alpha)}\left|h\left(s, y_{n}\left(p^{\alpha} s\right)\right)-h\left(s, y\left(p^{\alpha} s\right)\right)\right| d_{p, q} s \\
& +\left|\zeta_{2}\right| \sigma_{2}(T) \int_{0}^{\eta_{2}} \frac{\left(\eta_{2}-q s\right)_{p, q}^{(\alpha-1)}}{p^{(\alpha)} \Gamma_{p, q}(\alpha-1)}\left|h\left(s, y_{n}\left(p^{\alpha} s\right)\right)-h\left(s, y\left(p^{\alpha} s\right)\right)\right| d_{p, q} s \\
& +\left|\beta_{2}\right| \sigma_{2}(T) \int_{0}^{T} \frac{(T-q s)_{p, q}^{(\alpha-1)}}{p^{(\alpha)} \Gamma_{p, q}(\alpha-1)}\left|h\left(s, y_{n}\left(p^{\alpha} s\right)\right)-h\left(s, y\left(p^{\alpha} s\right)\right)\right| d_{p, q} s \\
& +\left|\gamma_{2}\right| \sigma_{2}(T) \int_{0}^{T / p} \frac{(T / p-q s)_{p, q}^{(\alpha-2)}}{p^{\left(\frac{\alpha}{2}\right)} \Gamma_{p, q}(\alpha-1)}\left|h\left(s, y_{n}\left(p^{\alpha} s\right)\right)-h\left(s, y\left(p^{\alpha} s\right)\right)\right| d_{p, q} s,
\end{aligned}
$$

which implies that

$$
\left\|\Psi y_{n}-\Psi y\right\| \rightarrow 0 \text { as } n \rightarrow \infty .
$$

Thus, the operator $\mathbb{F}$ is continuous.

Next, we show that $\Psi$ maps a bounded set into a bounded set in $\mathbb{C}([0, T], \mathbb{R})$. For a positive number $r>0$, let $B_{r}=\{y \in \mathbb{C}([0, T]):\|y\| \leq r\}$. Then, for any $y \in B_{r}$, we have

$$
\begin{aligned}
& (\Psi y)(t)=\int_{0}^{t} \frac{(t-q s)_{p, q}^{(\alpha-1)}}{p^{(\alpha)} \Gamma_{p, q}^{\alpha}(\alpha)} h\left(s, y\left(p^{\alpha-1} s\right)\right) d_{p, q} s \\
& +\frac{\zeta_{1}}{\Delta}\left[\left(\beta_{2}-\zeta_{2}\right) t-\left(\beta_{2} T+\gamma_{2}-\zeta_{2} \eta_{2}\right)\right] \int_{0}^{\eta_{1}} \frac{\left(\eta_{1}-q s\right)_{p, q}^{(\alpha-1)}}{p^{(\alpha)} \Gamma_{p, q}(\alpha)} h\left(s, y\left(p^{\alpha-1} s\right)\right) d_{p, q} s \\
& +\frac{\zeta_{2}}{\Delta}\left[\left(\gamma_{1}-\zeta_{1} \eta_{1}\right)-\left(\beta_{1}-\zeta_{1}\right) t\right] \int_{0}^{\eta_{2}} \frac{\left(\eta_{2}-q s\right)_{p, q}^{(\alpha-1)}}{p^{(\alpha)} \Gamma_{p, q}(\alpha-1)} h\left(s, y\left(p^{\alpha-1} s\right)\right) d_{p, q} s \\
& +\frac{\beta_{2}}{\Delta}\left[\left(\beta_{1}-\zeta_{1}\right) t-\left(\gamma_{1}-\zeta_{1} \eta_{1}\right)\right] \int_{0}^{T} \frac{(T-q s)_{p, q}^{(\alpha-1)}}{p^{\left(\begin{array}{c}
\alpha \\
2
\end{array}\right)} \Gamma_{p, q}(\alpha-1)} f\left(s, y\left(p^{\alpha-1} s\right)\right) d_{p, q} s \\
& +\frac{\gamma_{2}}{\Delta}\left[\left(\beta_{1}-\zeta_{1}\right) t-\left(\gamma_{1}-\zeta_{1} \eta_{1}\right)\right] \int_{0}^{T / p} \frac{(T / p-q s)_{p, q}^{(\alpha-2)}}{\left.p^{(\alpha-1}\right) \Gamma_{p, q}(\alpha-1)} f\left(s, y\left(p^{\alpha-2} s\right)\right) d_{p, q} s \\
& +\frac{1}{|\Delta|}\left[\alpha_{1} \beta_{2} t+\left|\beta_{1}\right|\left|\beta_{2}\right|\right] \int_{0}^{T / p} \frac{(T / p-q s)_{p, q}^{(\alpha-2)}}{p^{\left(\alpha_{2}^{\alpha-1}\right)} \Gamma_{p, q}(\alpha-1)}\left|h\left(s, y\left(p^{\alpha} s\right)\right)\right| d_{p, q} s
\end{aligned}
$$




$$
\begin{aligned}
& \leq \int_{0}^{T} \frac{(T-q s)^{(\alpha-1)}}{p^{(\alpha)} \Gamma_{p, q}(\alpha)}\left[p_{1}\left(\frac{s}{p^{\alpha-1}}\right) \psi(\|y\|)+p_{2}\left(\frac{s}{p^{\alpha-1}}\right)\right] d_{p, q} S \\
& +\left|\zeta_{1}\right| \sigma_{1}(T) \int_{0}^{\eta_{1}} \frac{\left(\eta_{1}-q s\right)_{p, q}^{(\alpha-1)}}{p^{\left(\begin{array}{c}
\alpha \\
2
\end{array}\right)} \Gamma_{p, q}(\alpha)}\left[p_{1}\left(\frac{s}{p^{\alpha-1}}\right) \psi(\|y\|)+p_{2}\left(\frac{s}{p^{\alpha-1}}\right)\right] d_{p, q} s \\
& +\left|\zeta_{2}\right| \sigma_{1}(T) \int_{0}^{\eta_{2}} \frac{\left(\eta_{2}-q s\right)_{p, q}^{(\alpha-1)}}{p^{\left(\begin{array}{c}
\alpha \\
2
\end{array}\right)} \Gamma_{p, q}(\alpha)}\left[p_{1}\left(\frac{s}{p^{\alpha-1}}\right) \psi(\|y\|)+p_{2}\left(\frac{s}{p^{\alpha-1}}\right)\right] d_{p, q} s \\
& +\left|\beta_{2}\right| \sigma_{2}(T) \int_{0}^{T} \frac{(T-q s)_{p, q}^{(\alpha-1)}}{p^{\left(\begin{array}{c}
\alpha \\
2
\end{array}\right)} \Gamma_{p, q}(\alpha)}\left[p_{1}\left(\frac{s}{p^{\alpha-1}}\right) \psi(\|y\|)+p_{2}\left(\frac{s}{p^{\alpha-1}}\right)\right] d_{p, q} S \\
& +\left|\gamma_{2}\right| \sigma_{2}(T) \int_{0}^{T / p} \frac{(T / p-q s)_{p, q}^{(\alpha-2)}}{p^{\left(\begin{array}{c}
\alpha-1 \\
2
\end{array}\right)} \Gamma_{p, q}(\alpha-1)}\left[p_{1}\left(\frac{s}{p^{\alpha-1}}\right) \psi(\|y\|)+p_{2}\left(\frac{s}{p^{\alpha-1}}\right)\right] d_{p, q} s \\
& \leq \psi(r)\left\{\int_{0}^{T} \frac{(T-q s)^{(\alpha-1)}}{p^{\left(\begin{array}{c}
\alpha \\
2
\end{array}\right)} \Gamma_{p, q}(\alpha)} p_{1}\left(\frac{s}{p^{\alpha-1}}\right) d_{p, q} s\right. \\
& +\left|\zeta_{1}\right| \sigma_{1}(T) \int_{0}^{\eta_{1}} \frac{(T-q s)_{p, q}^{(\alpha-1)}}{p^{\left(\begin{array}{c}
\alpha \\
2
\end{array}\right)} \Gamma_{p, q}(\alpha)} p_{1}\left(\frac{s}{p^{\alpha-1}}\right) d_{p, q} s \\
& +\left|\zeta_{2}\right| \sigma_{2}(T) \int_{0}^{\eta_{2}} \frac{\left(\eta_{2}-q s\right)_{p, q}^{(\alpha-1)}}{p^{\left(\begin{array}{c}
\alpha \\
2
\end{array}\right)} \Gamma_{p, q}(\alpha)} p_{1}\left(\frac{s}{p^{\alpha-1}}\right) d_{p, q} s \\
& +\left|\beta_{2}\right| \sigma_{2}(T) \int_{0}^{T} \frac{(T-q s)_{p, q}^{(\alpha-1)}}{p^{\left(\begin{array}{c}
\alpha \\
2
\end{array}\right)} \Gamma_{p, q}(\alpha)} p_{1}\left(\frac{s}{p^{\alpha-1}}\right) d_{p, q} s \\
& \left.+\left|\gamma_{2}\right| \sigma_{2}(T) \int_{0}^{T / p} \frac{(T / p-q s)_{p, q}^{(\alpha-2)}}{p^{(\alpha-1)} \Gamma_{p, q}(\alpha-1)} p_{1}\left(\frac{s}{p^{\alpha-1}}\right) d_{p, q} s\right\} \\
& +\left\{\int_{0}^{T} \frac{(T-q s)^{(\alpha-1)}}{p^{\left(\begin{array}{c}
\alpha \\
2
\end{array}\right)} \Gamma_{p, q}(\alpha)} p_{1}\left(\frac{s}{p^{\alpha-1}}\right) d_{p, q} s\right. \\
& +\left|\zeta_{1}\right| \sigma_{1}(T) \int_{0}^{\eta_{1}} \frac{\left(\eta_{1}-q s\right)_{p, q}^{(\alpha-1)}}{p^{\left(\begin{array}{c}
\alpha \\
2
\end{array}\right)} \Gamma_{p, q}(\alpha)} p_{1}\left(\frac{s}{p^{\alpha-1}}\right) d_{p, q} \mathcal{S} \\
& +\left|\zeta_{2}\right| \sigma_{2}(T) \int_{0}^{\eta_{2}} \frac{\left(\eta_{2}-q s\right)_{p, q}^{(\alpha-1)}}{p^{\left(\begin{array}{c}
\alpha \\
2
\end{array}\right)} \Gamma_{p, q}(\alpha)} p_{1}\left(\frac{s}{p^{\alpha-1}}\right) d_{p, q} s \\
& +\left|\beta_{2}\right| \sigma_{2}(T) \int_{0}^{T} \frac{(T-q s)_{p, q}^{(\alpha-1)}}{p^{\left(\begin{array}{c}
\alpha \\
2
\end{array}\right)} \Gamma_{p, q}(\alpha)} p_{1}\left(\frac{s}{p^{\alpha-1}}\right) d_{p, q} S \\
& \left.+\left|\gamma_{2}\right| \sigma_{2}(T) \int_{0}^{T / p} \frac{(T / p-q s)_{p, q}^{(\alpha-2)}}{p^{\left({ }^{\alpha-1}\right)} \Gamma_{p, q}(\alpha-1)} p_{1}\left(\frac{s}{p^{\alpha-1}}\right) d_{p, q} s\right\} .
\end{aligned}
$$

We have

$$
\|\Psi y\| \leq \psi(r) \omega_{1}+\omega_{2} \leq M .
$$

Therefore, the set $\Psi B_{r}$ is uniformly bounded.

Now, we show that $\Psi$ maps bounded sets into equicontinuous sets of $\mathbb{C}([0, T], \mathbb{R})$. Let $t_{1}, t_{2} \in[0, T]$ with $t_{1}<t_{2}$ be two points and $B_{r}$ be a bounded ball in $\Psi$. Then, for any $y \in B_{r}$, we obtain 


$$
\begin{aligned}
& \left|(\Psi y)\left(t_{2}\right)-(\Psi y)\left(t_{1}\right)\right| \\
& \leq\left|\int_{0}^{t_{2}} \frac{\left(t_{2}-q s\right)^{(\alpha-1)}}{p^{\left(\begin{array}{c}
\alpha \\
2
\end{array}\right)} \Gamma_{p, q}(\alpha)}\right| h\left(s, y\left(p^{\alpha-1} s\right)\right) \mid d_{p, q} s \\
& -\int_{0}^{t_{1}} \frac{\left(t_{1}-q s\right)^{(\alpha-1)}}{p^{\left(\begin{array}{c}
\alpha \\
2
\end{array}\right)} \Gamma_{p, q}(\alpha)}\left|h\left(s, y\left(p^{\alpha-1} s\right)\right)\right| d_{p, q} s \mid \\
& +\frac{1}{|\Delta|}\left|\zeta_{1}\left(\beta_{2}-\zeta_{2}\right)\right|\left(t_{2}-t_{1}\right) \int_{0}^{\eta_{1}} \frac{\left(\eta_{1}-q s\right)_{p, q}^{(\alpha-1)}}{p^{\left(\begin{array}{c}
\alpha \\
2
\end{array}\right)} \Gamma_{p, q}(\alpha)}\left|h\left(s, y\left(p^{\alpha-1} s\right)\right)\right| d_{p, q} s \\
& +\frac{1}{|\Delta|}\left|\alpha_{1}\right|\left|\alpha_{2}\right|\left(t_{2}-t_{1}\right) \int_{0}^{T} \frac{(T-q s)_{p, q}^{(\alpha-1)}}{p^{\left(\begin{array}{c}
\alpha \\
2
\end{array}\right)} \Gamma_{p, q}(\alpha)}\left|h\left(s, y\left(p^{\alpha-1} s\right)\right)\right| d_{p, q} s \\
& +\frac{1}{|\Delta|}\left|\alpha_{1}\right|\left|\alpha_{2}\right|\left(t_{2}-t_{1}\right) \int_{0}^{T} \frac{(T-q s)_{p, q}^{(\alpha-1)}}{p^{\left(\begin{array}{c}
\alpha \\
2
\end{array}\right)} \Gamma_{p, q}(\alpha)}\left|h\left(s, y\left(p^{\alpha-1} s\right)\right)\right| d_{p, q} s \\
& +\frac{1}{|\Delta|}\left|\alpha_{1} \beta_{2}\right|\left(t_{2}-t_{1}\right) \int_{0}^{T / p} \frac{(T / p-q s)_{p, q}^{(\alpha-2)}}{p^{\left(\begin{array}{c}
\alpha-1 \\
2
\end{array}\right)} \Gamma_{p, q}(\alpha-1)}\left|h\left(s, y\left(p^{\alpha} s\right)\right)\right| d_{p, q} s \\
& \leq \mid \int_{0}^{t_{1}}\left[\frac{\left(t_{2}-q s\right)^{(\alpha-1)}}{p^{\left(\begin{array}{c}
\alpha \\
2
\end{array}\right)} \Gamma_{p, q}(\alpha)}-\frac{\left(t_{1}-q s\right)^{(\alpha-1)}}{p^{\left(\begin{array}{c}
\alpha \\
2
\end{array}\right)} \Gamma_{p, q}(\alpha)}\right]\left[p_{1}\left(\frac{s}{p^{\alpha-1}}\right) \psi(r)+p_{2}\left(\frac{s}{p^{\alpha-1}}\right)\right] d_{p, q} s \\
& +\int_{t_{1}}^{t_{2}} \frac{\left(t_{1}-q s\right)^{(\alpha-1)}}{p^{\left(\begin{array}{c}
\alpha \\
2
\end{array}\right)} \Gamma_{p, q}(\alpha)}\left[p_{1}\left(\frac{s}{p^{\alpha-1}}\right) \psi(r)+p_{2}\left(\frac{s}{p^{\alpha-1}}\right)\right] d_{p, q} s \mid \\
& +\frac{1}{|\Delta|}\left|\alpha_{1}\right|\left|\alpha_{2}\right|\left(t_{2}-t_{1}\right) \int_{0}^{T} \frac{(T-q s)_{p, q}^{(\alpha-1)}}{p^{\left(\begin{array}{c}
\alpha \\
2
\end{array}\right)} \Gamma_{p, q}(\alpha)}\left[p_{1}\left(\frac{s}{p^{\alpha-1}}\right) \psi(r)+p_{2}\left(\frac{s}{p^{\alpha-1}}\right)\right] d_{p, q} S \\
& +\frac{1}{|\Delta|}\left|\alpha_{1}\right|\left|\alpha_{2}\right|\left(t_{2}-t_{1}\right) \int_{0}^{T} \frac{(T-q s)_{p, q}^{(\alpha-1)}}{p^{\left(\begin{array}{c}
\alpha \\
2
\end{array}\right)} \Gamma_{p, q}(\alpha)}\left[p_{1}\left(\frac{s}{p^{\alpha-1}}\right) \psi(r)+p_{2}\left(\frac{s}{p^{\alpha-1}}\right)\right] d_{p, q} s \\
& +\frac{1}{|\Delta|}\left|\alpha_{1}\right|\left|\alpha_{2}\right|\left(t_{2}-t_{1}\right) \int_{0}^{T} \frac{(T-q s)_{p, q}^{(\alpha-1)}}{p^{\left(\begin{array}{c}
\alpha \\
2
\end{array}\right)} \Gamma_{p, q}(\alpha)}\left[p_{1}\left(\frac{s}{p^{\alpha-1}}\right) \psi(r)+p_{2}\left(\frac{s}{p^{\alpha-1}}\right)\right] d_{p, q} s \\
& +\frac{1}{|\Delta|}\left|\alpha_{1} \beta_{2}\right|\left(t_{2}-t_{1}\right) \int_{0}^{T / p} \frac{(T / p-q s)_{p, q}^{(\alpha-2)}}{p^{\left(\frac{\alpha-1}{2}\right)} \Gamma_{p, q}(\alpha-1)}\left[p_{1}\left(\frac{s}{p^{\alpha-1}}\right) \psi(r)+p_{2}\left(\frac{s}{p^{\alpha-1}}\right)\right] d_{p, q} s .
\end{aligned}
$$

Obviously, the right-hand side of the above inequality tends to zero independently of $x \in B_{r}$ as $t_{2} \rightarrow t_{1}$. Thus, it follows by the Arzelá-Ascoli theorem that $\Psi: \mathbb{C}([0, T], \mathbb{R}) \rightarrow$ $\mathbb{C}([0, T], \mathbb{R})$ is completely continuous.

Now, the operator $\Psi$ satisfies all the conditions of Lemma 6 and, therefore, by its conclusion, either condition ( $i$ ) or condition (ii) holds.

Now, we show that the conclusion (ii) is not possible. Let

$$
\mathcal{U}=\{y \in \mathbb{C}([0, T], \mathbb{R}):\|y\| \leq M\}
$$

with $\psi(M) \omega_{1}+\omega_{2}<M$. Then, it can be shown that 


$$
\begin{aligned}
& |\Psi y| \leq \psi(\|y\|)\left\{\int_{0}^{T} \frac{(T-q s)^{(\alpha-1)}}{p^{\left(\begin{array}{c}
\alpha \\
2
\end{array}\right)} \Gamma_{p, q}(\alpha)} p_{1}\left(\frac{s}{p^{\alpha-1}}\right) d_{p, q} S\right. \\
& +\left|\zeta_{1}\right| \sigma_{1}(T) \int_{0}^{\eta_{1}} \frac{\left(\eta_{1}-q s\right)_{p, q}^{(\alpha-1)}}{p^{(\alpha)} \Gamma_{p, q}^{(\alpha)}(\alpha)} p_{1}\left(\frac{s}{p^{\alpha-1}}\right) d_{p, q} S \\
& +\left|\zeta_{2}\right| \sigma_{2}(T) \int_{0}^{\eta_{2}} \frac{\left(\eta_{2}-q s\right)_{p, q}^{(\alpha-1)}}{p^{(\alpha)} \begin{array}{c}
(\alpha) \\
\Gamma_{p, q}(\alpha)
\end{array}} p_{1}\left(\frac{s}{p^{\alpha-1}}\right) d_{p, q} s \\
& +\left|\beta_{2}\right| \sigma_{2}(T) \int_{0}^{T} \frac{(T-q s)_{p, q}^{(\alpha-1)}}{p^{(\alpha)} \Gamma_{p, q}^{(\alpha)}(\alpha)} p_{1}\left(\frac{s}{p^{\alpha-1}}\right) d_{p, q} S \\
& \left.+\left|\gamma_{2}\right| \sigma_{2}(T) \int_{0}^{T / p} \frac{(T / p-q s)_{p, q}^{(\alpha-2)}}{p^{\left(\frac{\alpha-1}{2}\right)} \Gamma_{p, q}(\alpha-1)} p_{2}\left(\frac{s}{p^{\alpha-1}}\right) d_{p, q} s\right\} \\
& +\left\{\int_{0}^{T} \frac{(T-q s)^{(\alpha-1)}}{p^{\left(\begin{array}{c}
\alpha \\
2
\end{array}\right)} \Gamma_{p, q}(\alpha)} p_{2}\left(\frac{s}{p^{\alpha-1}}\right) d_{p, q} s\right. \\
& +\left|\zeta_{1}\right| \sigma_{1}(T) \int_{0}^{\eta_{1}} \frac{\left(\eta_{1}-q s\right)_{p, q}^{(\alpha-1)}}{p^{(\alpha)} \Gamma_{p, q}(\alpha)} p_{2}\left(\frac{s}{p^{\alpha-1}}\right) d_{p, q} s \\
& +\left|\zeta_{2}\right| \sigma_{2}(T) \int_{0}^{\eta_{2}} \frac{\left(\eta_{2}-q s\right)_{p, q}^{(\alpha-1)}}{p^{(\alpha)} \Gamma_{p, q}(\alpha)} p_{2}\left(\frac{s}{p^{\alpha-1}}\right) d_{p, q} s \\
& +\left|\beta_{2}\right| \sigma_{2}(T) \int_{0}^{T} \frac{(T-q s)_{p, q}^{(\alpha-1)}}{p^{\left(\begin{array}{c}
\alpha \\
2
\end{array}\right)} \Gamma_{p, q}(\alpha)} p_{2}\left(\frac{s}{p^{\alpha-1}}\right) d_{p, q} S \\
& \left.+\left|\gamma_{2}\right| \sigma_{2} \mid(T) \int_{0}^{T / p} \frac{(T / p-q s)_{p, q}^{(\alpha-2)}}{p^{(\alpha-1)} \Gamma_{p, q}(\alpha-1)} p_{2}\left(\frac{s}{p^{\alpha-1}}\right) d_{p, q} s\right\} \\
& \leq \psi(M) \omega_{1}+\omega_{2} \leq M .
\end{aligned}
$$

Suppose there exists $y \in \partial \mathcal{U}$ and $\lambda \in(0,1)$ such that $y=\lambda \Psi x$. Then, for such choices of $x$ and $\lambda$, we have

$$
M=\|y\|=\lambda\|\Psi y\|<\psi(\|y\|) \omega_{1}+\omega_{2}<M .
$$

This leads to a contradiction. Accordingly, by Lemma 6, we have that $\Psi$ has a fixed point $y \in \overline{\mathcal{U}}$, which is a solution of the boundary value problem (4). Therefore, the proof is completed.

Remark 3. If $p_{1}, p_{2}$ in $\left(A_{4}\right)$ are continuous, then $\omega_{i} \leq A\left\|p_{i}\right\|, i=1,2$, where $A$ is defined by (23).

\section{Examples}

In this section, we give some examples to illustrate the investigated results.

Example 1. Consider the following fractional $(p, q)$-difference equation with the boundary value problem

$$
\left\{\begin{array}{l}
{ }^{c} D_{1 / 4,1 / 5}^{3 / 2} y(t)=\frac{\lambda}{2}\left(\sin (t)+\tan ^{-1}(y(t / 8))+y(t / 8)\right), t \in[0,8], \\
y(0)+\frac{1}{2} D_{p, q} y(0)=y(1 / 3), \quad \frac{1}{2} y(1)+\frac{3}{2} D_{p, q} y(4)=y(2 / 3) .
\end{array}\right.
$$

By applying Theorem 3 with $p=1 / 4, q=1 / 5, \alpha=3 / 2, \beta_{1}=1, \gamma_{1}=1 / 2, \zeta_{1}=1$, $\beta_{2}=1 / 2, \gamma_{2}=3 / 2, \zeta_{2}=1, \eta_{1}=1 / 3, \eta_{2}=2 / 3, T=1$, and $\lambda$ is a constant to be fixed later on. By simple calculation, we have $\Delta=1 / 12, \sigma_{1}(1)=4 / 3$, and $\sigma_{2}(1)=2$. Let 


$$
\lambda<\left[\frac{1}{\Gamma_{1 / 4,1 / 5}(3 / 2)}\left(\frac{171 \sqrt{30}+8 \sqrt{15}+48 \sqrt{5}-216 \sqrt{6}}{45 \sqrt{30}-72 \sqrt{6}}\right)\right]^{-1} .
$$

It is not difficult to show that

$$
|f(t, y)-f(t, z)| \leq \lambda|y-z|,
$$

for each $t \in[0,8]$, and

$$
k=\frac{\lambda}{\Gamma_{1 / 4,1 / 5}(3 / 2)}\left(\frac{171 \sqrt{30}+8 \sqrt{15}+48 \sqrt{5}-216 \sqrt{6}}{45 \sqrt{30}-72 \sqrt{6}}\right)<1 .
$$

Then, all the assumptions of Theorem 3 are satisfied. Accordingly, by Theorem 3, the boundary value problem (28) has a unique solution.

Example 2. Consider the following fractional $(p, q)$-difference equation with the boundary value problem

$$
\left\{\begin{array}{l}
{ }^{c} D_{1 / 4,1 / 5}^{3 / 2} y(t)=\frac{1}{16} \cos t^{2} \sin \left(\frac{|y(t / 8)|}{2}\right)+\frac{e^{-y(t / 8)}\left(t^{2}+1\right)}{2+t^{2}}+\frac{1}{3}, t \in[0,8], \\
y(0)+\frac{1}{2} D_{p, q} y(0)=y(1 / 3), \quad \frac{1}{2} y(1)+\frac{3}{2} D_{p, q} y(4)=y(2 / 3) .
\end{array}\right.
$$

By applying Theorem 5 with $p=1 / 4, q=1 / 5, \alpha=3 / 2, \beta_{1}=1, \gamma_{1}=1 / 2, \zeta_{1}=$ $1, \beta_{2}=1 / 2, \gamma_{2}=3 / 2, \zeta_{2}=1, \eta_{1}=1 / 3, \eta_{2}=2 / 3, T=1$. By simple calculation, we have $\Delta=1 / 12, \sigma_{1}(1)=4 / 3$, and $\sigma_{2}(1)=2$.

On the other hand, we have

$$
\begin{aligned}
|f(t, y)| & =\left|\frac{1}{16} \cos t^{2}\right| \sin (|y| / 2 \mid)+\frac{e^{-y}\left(t^{2}+1\right)}{2+t^{2}}+\frac{1}{3} \mid \\
& \leq \frac{1}{32}|y|+1 .
\end{aligned}
$$

Obviously, $p_{1}=1 / 16, p_{2}=1, \psi(M)=M$. By simple computation, we obtain $\omega_{1} \approx 0.9149007474, \omega_{2} \approx 29.27682392$. Additionally, from the condition (27), it follows that $M>344.0315046$. Thus, all assumptions of Theorem 5 are satisfied. Therefore, the boundary value problem (29) has at least one solution.

\section{Conclusions}

In this paper, we study nonlocal fractional $(p, q)$-difference equations with separated nonlocal boundary conditions. The existence of solutions for the problem is given by applying some well-known tools in fixed-point theory, such as Banach's contraction mapping principle, Krasnoselskii's fixed-point theorem, and the Leray-Schauder nonlinear alternative. Some illustrating examples are also presented. We hope that the paper will inspire interested readers working in this field to draw upon these ideas and techniques.

Author Contributions: Conceptualization, P.N., K.N., J.T., S.K.N. and B.A.; investigation, P.N., K.N., J.T., S.K.N. and B.A.; methodology, P.N., K.N., J.T., S.K.N. and B.A.; validation, P.N., K.N., J.T., S.K.N. and B.A.; visualization, P.N., K.N., J.T., S.K.N. and B.A.; writing-original draft, P.N. and K.N.; writing-review and editing, P.N. and K.N. All authors have read and agreed to the published version of the manuscript.

Funding: This research received no external funding.

Institutional Review Board Statement: Not applicable.

Informed Consent Statement: Not applicable.

Data Availability Statement: Not applicable. 
Acknowledgments: This research has received funding support from the National Science, Research and Innovation Fund (NSRF), Thailand.

Conflicts of Interest: The authors declare no conflict of interest.

\section{References}

1. Agarwal, R.P.; Belmekki, M.; Benchohra, M. A survey on semilinear differential equations and inclusions involving RiemannLiouville fractional derivative. Adv. Differ. Equ. 2009, 2009, 981728. [CrossRef]

2. Gafiychuk, V.; Datsko, B.; Meleshko, V. Mathematical modeling of different types of instabilities in time fractional reactiondiffusion system. Comput. Math. Appl. 2010, 59, 101-1107. [CrossRef]

3. Ahmad, B.; Agarwal. R.P. On nonlocal fractional boundary value problems. Dyn. Contin. Discrete Impuls. Syst. 2011, 18, 535-544.

4. Baleanu, D.; Mustafa, O.G.; O'Regan, D. A Nagumo-like uniqueness theorem for fractional differential equations. J. Phys. Math. Theor. 2011, 44, 39. [CrossRef]

5. Wang, Y.; Liu, L.; Wu, Y. Positive solutions for a nonlocal fractional differential equation. Nonlinear. Anal. 2011, 74, 3599-3605. [CrossRef]

6. Ford, N.J.; Morgado, M.L. Fractional boundary value problems: analysis and numerical methods. Fract. Calc. Appl. Anal. 2011, 14, 554-567. [CrossRef]

7. Ahmad, B.; Nieto, J.J.; Alsaidi, A.; El-Shahed, M. A study nonlinear Langevin equation involving two fractional orders in different interval. Nonlinear. Anal. 2012, 13, 599-606. [CrossRef]

8. Ahmad, B.; Ntouyas, S.K. Nonlinear fractional differential equations and inclusions of arbitrary order and multi-strip boundary conditions. Electron. J. Differ. Equ. 2012, 2012, 98.

9. Chen, F.; Nieto, J.J.; Zhou, Y. Global attractivity for nonlinear fractional differential equations. Nonlinear. Anal. 2012, 13, 287-298. [CrossRef]

10. Kiethelm, K. The Analysis of Fractional Differential Equation; Springer: New York, NY, USA, 2010.

11. Kilbas, A.A.; Srivastava, H.M.; Trujillo, H.J. Theory and Application of the Fractional Differential Equations. In North-Holland Mathematics Studies; Elsevier: Amsterdam, The Netherlands, 2006; Volume 204.

12. Lakshmikantham, V.; Leela, S.; Devi.; J.V. Theory of Fractional Dynamic Systyms; Cambridge Scientific Publishers: Cambridge, UK, 2009.

13. Miller, K.S.; Ross, B. An Introduction to the Fractional Calculus and Differential Equations; Wiley-Interscience: New York, NY, USA, 1993.

14. Podlubny, I. Fractional Differential Equations; Accademic Press: New York, NY, USA, 1999.

15. Samko, S.G.; Kilbas, A.A.; Marichev, O.I. Fractional Integrals and Derivatives; Gordon and Breach Science: Yverdon, Switzerland, 1993.

16. Ahmad, B.; Alsaedi, A.; Ntouyas, S.K.; Tariboon, J. Hadamard-Type Fractional Differential Equations. In Inclusions and Inequalities; Springer: Cham, Switzerland, 2017.

17. Zhou, Y. Basic Theory of Fractional Differential Equations; World Scientific Publishing Company: Singapore, 2014.

18. Jackson, F.H. On a q-definite integrals. Quart. J. Pure Appl. Math. 1910, 41, 193-203.

19. Jackson, F.H. q-Difference equations. Am. J. Math. 1910, 32, 305-314. [CrossRef]

20. Fock, V. Zur theorie des wasserstoffatoms. Z. Physik 1935, 98, 145-154. [CrossRef]

21. Bangerezaka, G. Variational q-calculus. J. Math. Anal. Appl. 2004, 289, 650-665. [CrossRef]

22. Asawasamrit, S.; Sudprasert, C.; Ntouyas, S.; Tariboon, J. Some results on quantum Hanh integral inequalities. J. Inequal. Appl. 2019, 2019, 154. [CrossRef]

23. Bangerezako, G. Variational calculus on $q$-nonuniform. J. Math. Anal. Appl. 2005, 306, 161-179. [CrossRef]

24. Exton, H. q-Hypergeometric Functions and Applications; Hastead Press: New York, NY, USA, 1983.

25. Annyby, H.M.; Mansour, S.K. q-fractional Calculus and Equations; Springer: Berlin/Helidelberg, Germany, 2012.

26. Ernst, T. A Comprehensive Treatment of q-Calculus; Springer: Basel, Swizterland, 2012.

27. Ernst, T. A History of q-Calculus and a New Method; UUDM Report 2000:16; Department of Mathematics, Uppsala University: Uppsala, Sweden, 2000.

28. Ferreira, R.A.C. Nontrivial solutions for fractional $q$-difference boundary value problems. Electron. J. Qual. Theory Differ. Equ. 2010, 2010, 70. [CrossRef]

29. Aslam, M.; Awan, M.U.; Noor, K.I. Quantum Ostrowski inequalities for $q$-differentiable convex function. J. Math. Inequal. 2016, 10, 1013-1018.

30. Aral, A.; Gupta, V.; Agarwal, R.P. Applications of q-Calculus in Operator Theory; Springer: New York, NY, USA, 2013.

31. Gauchman, H. Integral inequalities in q-calculus. J. Comput. Appl. Math. 2002, 47, 281-300. [CrossRef]

32. Kunt, M.; Aljasem, M. Riemann-Liouville fractional quantum Hermite-Hadamard type inequalities for convex functions. Konuralp J. Math. 2020, 8, 122-136.

33. Dobrogowska, A.; Odzijewicz, A. A second order $q$-difference equation solvable by factorization method. J. Comput. Appl. Math. 2006, 193, 319-346. [CrossRef]

34. Gasper, G.; Rahman, M. Some systems of multivariable orthogonal q-Racah polynomials. Ramanujan J. 2007, 13, $389-405$. [CrossRef] 
35. Ismail, M.E.H.; Simeonov, P. q-Difference operators for orthogonal polynomials. J. Comput. Appl. Math. 2009, $233,749-761$. [CrossRef]

36. Bohner, M.; Guseinov, G.S. The $h$-Laplace and $q$-Laplace transforms. J. Comput. Appl. Math. 2010, 365, 75-92. [CrossRef]

37. El-Shahed, M.; Hassan, H.A. Positive solutions of $q$-difference equation. Proc. Amer. Math. Soc. 2010, 138, 1733-1738. [CrossRef]

38. Ahmad, B. Boundary-value problems for nonlinear third-order $q$-difference equations. Electron. J. Differ. Equ. 2011, $2011,94$.

39. Ahmad, B.; Alsaedi, A.; Ntouyas, S.K. A study of second-order $q$-difference equations with boundary conditions. Adv. Differ. Equ. 2012, 2012, 35. [CrossRef]

40. Ahmad, B.; Nieto, J.J. On nonlocal boundary value problems of nonlinear $q$-difference equation. Adv. Differ. Equ. 2012, $2012,81$. [CrossRef]

41. Kac,V.; Cheung, P. Quantum Calculus; Springer: New York, NY, USA, 2002.

42. Al-Salam, W.A. Some fractional $q$-integrals and $q$-derivatives. Proc. Edinburgh Math. Soc. 1967, 15, 135-140. [CrossRef]

43. Agarwal, R.P. Certain fractional $q$-integrals and $q$-derivatives. Math. Proc. Camb. Philos. Soc. 1969, 66, 365-370. [CrossRef]

44. Ahmad, B.; Ntouyas, S.K.; Purnaras, I.K. Existence results for nonlinear $q$-difference equations with nonlocal boundary conditions. Comm. Appl. Nonlinear Anal. 2012, 19, 59-72.

45. El-Shahed, M.; Al-Askar, F. Positive solutions for boundary value problem of nonlinear fractional $q$-difference equations. ISRN Math. Anal. 2011, 2011, 385459. [CrossRef]

46. Ferreira, R.A.C. Positive solutions for a class of boundary value problems with fractional $q$-difference equations. Comput. Math. Appl. 2011, 61, 367-373. [CrossRef]

47. Graef, J.R.; Kong, L. Positive solutions for a class of higher order boundary value problems with fractional $q$-derivative. Appl. Math. Comput. 2012, 218, 9682-9689. [CrossRef]

48. Ma, J.; Yang, J. Existence of solutions for multi-point boundary value problem of fractional q-difference equation. Appl. Math. Comput. 2011, 2011, 92. [CrossRef]

49. Nuntigrangjana, T.; Putjuso, S.; Ntouyas, S.K.; Tariboon, J. Impulsive quantum $(p, q)$-difference equations. Adv. Differ. Equ. 2020, 2020, 98.

50. Chakrabarti, R.; Jagannathan, R. A $(p, q)$-oscillator realization of two-parameter quantum algebras. J. Phys. A Math. Gen. 1991, 24, L711-L718. [CrossRef]

51. Burban, I. Two-parameter deformation of the oscillator algebra and $(p, q)$-analog of two-dimensional conformal field theory. J. Nonlinear Math. Phys. 1995, 2, 384-391. [CrossRef]

52. Burban, I.M.; Klimyk, A.U. $(p, q)$-differentiation, $(p, q)$-integration, and $(p, q)$-hypergeometric functions related to quantum groups. Integral Transform. Spec. Funct. 1994, 2, 15-36. [CrossRef]

53. Hounkonnou, M.N.; Désiré, J.; Kyemba, B.R. $(p, q)$-calculus: Differentiation and integration. SUT J. Math. 2013, $49,145-167$.

54. Aral, A.; Gupta, V. Applications of $(p, q)$-gamma function to Szász durrmeyer operators. Publ. de L'Institute Math. 2017, 102, 211-220. [CrossRef]

55. Sahai, V.; Yadav, S. Representations of two parameter quantum algebras and $(p, q)$-special functions. Adv. Differ. Equ. 2007, 335, 268-279.

56. Sadjang, P.N. On the fundamental theorem of $(p, q)$-calculus and some (p.q)-taylor formulas. Results Math. 2018, 73, 39. [CrossRef]

57. Usman, T.; Saif.; M.; Choi, J. Certain identities associated with $(p, q)$-binomial coefficients and $(p, q)$-Stirling polynomials of the second kind. Symmetry. 2020, 12, 1436. [CrossRef]

58. Sadjang, P.N. On the $(p, q)$-gamma and the $(p, q)$-beta functions. arXiv 2015, arXiv:1506.07394.

59. Sadjang, P.N. On two $(p, q)$-analogues of the laplace transform. J. Differ. Equ. Appl. 2017, 23, 1562-1583.

60. Mursaleen, M.; Ansari, K.J.; Khan, A. On $(p, q)$-analogues of Bernstein operators. Appl. Math. Comput. 2015, $266,874-882$. [CrossRef]

61. Mursaleen, M.; Ansari, K.J.; Khan, A. Erratum to “On $(p, q)$-analogues of Bernstein operators.” Appl. Math. Comput. 2016, 278, 70-71. [CrossRef]

62. Kang, S.M.; Rafiq, A.; Acu, A.M.; Ali, F.; Kwun, Y.C. Erratum to "Some approximation properties of $(p, q)$-Bernstein operators". J. Inequal. Appl. 2016, 2016, 169. [CrossRef]

63. Mursaleen, M.; Khan, F.; Khan, A. Approximation by $(p, q)$-Lorentz polynomials on a compact disk. Complex Anal. Oper. Theory. 2016, 10, 1725-1740. [CrossRef]

64. Cai, Q.-B.; Zhou, G. On $(p, q)$-analogues of Kantorovich type Bernstein-Stancu-Schurer operator. Appl. Math. Comput. 2016, 276, 12-20. [CrossRef]

65. Mursaleen, M.; Ansari, K.J.; Khan, A. Some approximation results of $(p, q)$-analogues of Bernstein-Stancu operators. Appl. Math. Comput. 2015, 264, 392-402. [CrossRef]

66. Acar, T.; Aral, A.; Mohiuddine, S.A. On Kantorovich modification of $(p, q)$-Baskakov operators. J. Inequal. Appl. 2016, 2016, 98. [CrossRef]

67. Mursaleen, M.; Nasiruzzaman, M.; Khan, F.; Khan, A. $(p, q)$-analogues of divided difference and Bernstein operators. J. Nonlinear Funct. Anal. 2017, 2017, 25.

68. Wachs, M.; White, D. ( $p, q)$-Stirling numbers and set partition statistics. J. Combin. Theory Ser. A. 1991, 56, 27-46. [CrossRef]

69. Wachs, M.L. $\sigma$-restricted growth functions and $(p, q)$-Stirling numbers. J. Combin. Theory Ser. A 1994, 68, 470-480. [CrossRef] 
70. Remmel, J.B.; Wachs, M. Rook theory, generalized Stirling numbers and $(p, q)$-analogues. Electron. J. Combin. 2004,11, R84. [CrossRef]

71. Médics, A.D.; Leroux, P. Generalized Stirling numbers, convolution formula and ( $p, q)$-analogues. Can. J. Math. 1995, 11, 474-499. [CrossRef]

72. Bukweli-Kyemba, J.D.; Hounkonnou, M.N. Quantum deformed algebra: Coherent states and special functions. arXiv 2013, arXiv:1301.0116v1.

73. Prabseang, J.; Nonlaopon, K.; Tariboon, J. $(p, q)$-Hermite-Hadamard inequalities for double integral and $(p, q)$-differentiable convex functions. Axioms 2019, 8, 68. [CrossRef]

74. Kalsoom, H.; Amer, M.; Junjua, M.D.; Hassain, S.; Shahxadi, G. $(p, q)$-estimates of Hermite-Hadamard-type inequalities for coordinated convex and quasi convex function. Mathematics 2019, 7, 683. [CrossRef]

75. Kamsrisuk, N.; Promsakon, C.; Ntouyas, S.K.; Tariboon, J. Nonlocal boundary value problems for $(p, q)$-difference equations. J. Differ. Equ. 2018, 10, 183-195. [CrossRef]

76. Promsakon, C.; Kamsrisuk, N.; Ntouyas, S.K.; Tariboon, J. On the second-order $(p, q)$-difference equation with separated boundary conditions. Adv. Math. Phys. 2018, 2018, 9089865. [CrossRef]

77. Soontharanon, J.; Sitthiwirattham, T. On fractional $(p, q)$-calculus. Adv. Differ. Equ. 2020, 2020, 35. [CrossRef]

78. Gradimir, V.M.; Vijay, G.N.M. ( $p, q)$-beta functions and applications in approximation. Bol. Soc. Mat. Mex. 2018, $24,219-237$.

79. Tun, M.; Gov, E. Some integral inequalities via $(p, q)$-calculus on finite intervals. RGMIA Res. Rep. Coll. $2016,19,95$.

80. Krasnoselskii, M.A. Two remarks on the method of successive approximations. Uspekhi Mat. Nauk. 1995, 10, $123-127$.

81. Granas, A.; Dugundji, J. Fixed Point Theory and Applications; Springer: New York, NY, USA, 2003. 